\title{
STABILITY AND CONVERGENCE ANALYSIS OF SECOND-ORDER SCHEMES FOR A DIFFUSE INTERFACE MODEL WITH PENG-ROBINSON EQUATION OF STATE*
}

\author{
Qiujin Peng \\ Institute for Mathematical Sciences, Renmin University of China, Beijing 100872, China \\ Email: pengqiujin@ruc.edu.cn \\ Zhonghua Qiao \\ Department of Applied Mathematics, The Hong Kong Polytechnic University, Hung Hom, Hong Kong \\ Email: zhonghua.qiao@polyu.edu.hk \\ Shuyu Sun \\ Division of Physical Sciences and Engineering, King Abdullah University of Science and Technology, \\ Thuwal 23955-6900, Kingdom of Saudi Arabia \\ Email: shuyu.sun@kaust.edu.sa
}

\begin{abstract}
In this paper, we present two second-order numerical schemes to solve the fourth order parabolic equation derived from a diffuse interface model with Peng-Robinson Equation of state (EOS) for pure substance. The mass conservation, energy decay property, unique solvability and $L^{\infty}$ convergence of these two schemes are proved. Numerical results demonstrate the good approximation of the fourth order equation and confirm reliability of these two schemes.
\end{abstract}

Mathematics subject classification: 65N06, 65B99.

Key words: Diffuse interface model, Fourth order parabolic equation, Energy stability, Convergence.

\section{Introduction}

Multi-phase fluid mixture and its behaviors play important roles in many natural and engineering systems, especially in subsurface petroleum reservoirs [5,6,30-33]. It remains a challenge to understand and to model the complex interaction between phases, namely the physically distinct, separable portions of substance. In the classical theory known as the sharp interface model $[9,27,39]$, an interface between two fluids is modeled as an infinitely thin, or sharp two-dimensional entity, and it is endowed with interface properties such as surface tension. In a more detailed continuum model called as the diffuse interface model, the sharp fluid-fluid interface is replaced by a small but finite-thickness layer in which the fluids may mix. Even though the concept of a diffuse interface was originally proposed a long time ago by van der Waals [36], its numerical simulation with realistic fluids has been investigated only in recent years $[20,22]$. The diffuse interface theory is also known as the gradient theory $[4,10]$ in the chemical society, or phase field theory [3] in the fluid dynamics society. Unlike sharp interface models where surface tension must be provided as an input parameter, diffuse interface models have been used to predict surface tension $[4,10,18,19]$. In particular, the surface tensions of petroleum fluids have been well predicted by diffuse interface models in one spatial dimension

\footnotetext{
* Received May 1, 2016 / Revised version received November 14, 2016 / Accepted November 28, 2016 /

Published online September 13, 2017 /
} 
together with Peng-Robinson Equation of State (EOS) [21], which is one of the most popular equations of state for hydrocarbon systems. However, little work has been carried out to investigate the numerical simulation of Peng-Robinson-EOS-equipped diffuse interface models in multiple spatial dimensions [22].

Inspired by the favorable properties of the Cahn-Hilliard equation and its derivation, we provide the fourth-order parabolic equation to describe the equilibrium state and the flow of the components in the crude oil in this article. Numerical experiments are our indispensable tools to investigate the solution to this equation. Previously proposed schemes for the CahnHilliard equation $[7,8,10-14,17,29,34,35,38]$ and other kinetics equations contain fourth order term $[23,25,26,28,37]$ could be used as valuable references. The main contribution of this work is to develop two second-order energy stable numeircal schemes for the two-dimensional diffuse interface model with Peng-Robinson EOS of single component substance. The mass conservation and unique solvability are proved. The energy stability of these two schemes are achieved following the approach in [24]. However, it is not that smooth as we expected to obtain the $L^{\infty}$ convergence of these two schemes attributed to the unboundedness of the free energy density of any given substance and its first and second order derivatives respect to the molar density. Taking the work of Li et al. [15] as reference, we overcome this difficulty with a nontrivial arguments.

The rest of this paper is organized as follows. In the second section, we present the mathematical model of the diffuse interface equation derived from the Peng-Robinson EOS and the scaled fourth order equation for multi-component substances and demonstrate its energy decreasing and mass conservation characters. In the third section, we present notations on the discrete space and some auxiliary lemmas. After that, the $L^{\infty}$ convergence of the CrankNicolson scheme and the second order linearized scheme will be demonstrated in the fourth and fifth sections, respectively. And then, we provide the numerical results of these two schemes and compare them with previously published ones. The conclusion of this article will be provided in the end.

\section{Mathematical Model of Fluid Systems with Diffuse Interface}

We consider a fluid system consisting of fixed species amount on a fixed domain with spatially uniform-distributed given temperature.

\subsection{Helmholtz free energy from Peng-Robinson EOS}

Let $M$ denote the number of components in the fluid mixture, $n_{i}$ represent the molar concentration of the component $i$, and

$$
\mathbf{n}=\left(n_{1}, n_{2}, \cdots, n_{M}\right)^{T}
$$

be the molar concentrations of all components and $n=n_{1}+n_{2}+\cdots+n_{M}$ the molar density of the fluid. According to the diffuse interface model, the total Holmholtz free energy has the following form,

$$
F(\mathbf{n})=\int_{\Omega} f(\mathbf{n}) d \mathbf{x}=\int_{\Omega} f_{0}(\mathbf{n}) d \mathbf{x}+\int_{\Omega} f_{\nabla}(\mathbf{n}) d \mathbf{x} .
$$

From Peng-Robinson EOS, the Helmholtz free energy $f_{0}(\mathbf{n})$ of a homogeneous fluid is given by

$$
f_{0}(\mathbf{n})=f_{0}^{\text {ideal }}(\mathbf{n})+f_{0}^{\text {excess }}(\mathbf{n}),
$$




$$
\begin{aligned}
& f_{0}^{\text {ideal }}(\mathbf{n})=R T \sum_{i=1}^{M} n_{i}\left(\ln n_{i}-1\right), \\
& f_{0}^{\text {excess }}(\mathbf{n})=-n R T \ln (1-b n)+\frac{a(T) n}{2 \sqrt{2} b} \ln \left(\frac{1+(1-\sqrt{2}) b n}{1+(1+\sqrt{2}) b n}\right) .
\end{aligned}
$$

Here $T$ reprents the temperature of the mixture and $R$ represents the universal gas constant (approximately $8.31432 \mathrm{JK}^{-1} \mathrm{~mol}^{-1}$ ). The energy parameter $a=a(T)$ and the covolume parameter $b$ are given by the following mixing rules,

$$
a(T)=\sum_{i=1}^{M} \sum_{j=1}^{M} y_{i} y_{j}\left(a_{i} a_{j}\right)^{1 / 2}\left(1-k_{i j}\right), \quad b=\sum_{i=1}^{M} y_{i} b_{i},
$$

where $y_{i}=n_{i} / n$ is the mole fraction of component $i$. The Peng-Robinson parameters $a_{i}$ and $b_{i}$ for pure-substance component $i$ can be computed from the critical properties of the species as follows,

$$
a_{i}=a_{i}(T)=0.45724 \frac{R^{2} T_{c_{i}}^{2}}{P_{c_{i}}}\left(1+m_{i}\left(1-\sqrt{\frac{T}{T_{c_{i}}}}\right)\right)^{2}, \quad b_{i}=0.07780 \frac{R T_{c_{i}}}{P_{c_{i}}} .
$$

The binary interaction coefficient $k_{i j}$ of Peng-Robinson EOS is usually computed from experimental correlation. The critical temperature $T_{c_{i}}$ and critical pressure $P_{c_{i}}$ of a pure substance are intrinsic properties of the species, and they are available for most species encountered in application. The parameter $m_{i}$ contained in the formula for $a_{i}$ is experimentally correlated to the accentric parameter $\omega_{i}$ of the species by the following equations:

$$
m_{i}= \begin{cases}0.37464+1.54226 \omega_{i}-0.26992 \omega_{i}^{2}, & \omega_{i} \leq 0.49, \\ 0.379642+1.485030 \omega_{i}-0.164423 \omega_{i}^{2}+0.016666 \omega_{i}^{3}, & \omega_{i}>0.49 .\end{cases}
$$

The gradient part of the free energy density $f_{\nabla}(\mathbf{n})$ is in the following form,

$$
f_{\nabla}(\mathbf{n})=\frac{1}{2} \sum_{i, j=1}^{M} c_{i j} \nabla n_{i} \cdot \nabla n_{j},
$$

where the influence parameter $c_{i j}$ is a function of temperature and molar concentrations which can also be provided by Peng-Robinson EOS in the mixing rule given by modified geometric mean as

$$
c_{i j}=\left(1-\beta_{i j}\right) \sqrt{c_{i} c_{j}},
$$

where the parameter $\beta_{i j}$ is the binary interaction coefficient for the influence parameter. Stability of the interface requires $\beta_{i j}$ to be included in the interval $[0,1]$ and $\beta_{i j}=\beta_{j i}$. For most systems, $\beta_{i j}$ is assumed to be zero. When $\beta_{i j}=0$, the mixing rule is reduced to the simple geometric mean. The influence parameter of pure substance $c_{i}$ is related to the Peng-Robinson parameters $a_{i}$ and $b_{i}$ by

$$
c_{i}=a_{i} b_{i}^{2 / 3}\left(m_{1, i}^{c}\left(1-\frac{T}{T_{c_{i}}}\right)+m_{2, i}^{c}\right),
$$

where $m_{1, i}^{c}$ and $m_{2, i}^{c}$ are the coefficients correlated merely with the accentric factor $\omega_{i}$ of the component $i$ by the following relations,

$$
m_{1, i}^{c}=-\frac{10^{-16}}{1.2326+1.3757 \omega_{i}}, \quad m_{2, i}^{c}=\frac{10^{-16}}{0.9051+1.5410 \omega_{i}} .
$$




\subsection{A single component two-phase system}

In this work, we would like to consider a single component two-phase system (i.e., the fluid being pure substance). In this case, the total Helmholtz free energy $F$ is reduced to

$$
F(n)=\int_{\Omega} f(n) d \mathbf{x}=\int_{\Omega}\left(f_{0}(n)+\frac{c}{2}|\nabla n|^{2}\right) d \mathbf{x} .
$$

The evolution of the molar concentration $n$ can be written under mass conservation form

$$
\frac{\partial n}{\partial t}=-\nabla \cdot J
$$

where $J$ is the mass flux which has the form

$$
J=-\nabla \frac{\delta f(n)}{\delta n} .
$$

Therefore, giving periodic boundary condition, we have the following governing equation for a single component two-phase system:

$$
\frac{\partial n(\mathbf{x}, t)}{\partial t}=-c \Delta^{2} n(\mathbf{x}, t)+\Delta \mu_{0}(n(\mathbf{x}, t)),
$$

subjecting to the initial condition

$$
n(\mathbf{x}, 0)=n_{0}(\mathbf{x}) .
$$

For the energy density $f_{0}(n(\mathbf{x}, t))$, we have [24]

$$
\frac{\partial f_{0}(n(\mathbf{x}, t))}{\partial t}=\frac{\partial f_{0}}{\partial n} \frac{\partial n}{\partial t}=\mu_{0}(n(\mathbf{x}, t)) \frac{\partial n(\mathbf{x}, t)}{\partial t},
$$

where the first order derivative of $f_{0}(n)$ with respect to the molar density $n(\mathbf{x}, t)$ means the homogeneous chemical potential of the substance, the detailed form of which is as follows,

$$
\mu_{0}(n)=R T \ln \left(\frac{n}{1-b n}\right)+\frac{R T b n}{1-b n}+\frac{a(T)}{2 \sqrt{2} b} \ln \left(\frac{1+(1-\sqrt{2}) b n}{1+(1+\sqrt{2}) b n}\right)-\frac{a(T) n}{1+2 b n-b^{2} n^{2}} .
$$

Lemma 2.1. (Mass conservation.) If $n(\mathbf{x}, t)$ is a solution of the fourth order equation (2.5a)(2.5b) under periodic boundary condition, then we can get the following mass conservation identity

$$
\frac{d}{d t} \int_{\Omega} n(\mathbf{x}, t) d x=0
$$

The proof of this lemma is trivial. It can be obtained by integrating both sides of the equation (2.5a) over the domain $\Omega$, using Green's theorem with the periodic boundary condition. Moreover, taking the inner product of $(2.5 \mathrm{a})$ with the term $c \Delta n(\mathbf{x}, t)-\mu_{0}(n(\mathbf{x}, t))$ under the periodic boundary condition, we can also obtain the following energy identity.

Lemma 2.2. (Energy identity.) If $n(\mathbf{x}, t)$ is a solution of the fourth-order equation (2.5a)(2.5b) under periodic boundary condition, the following energy identity can be guaranteed

$$
\frac{d F(n(\mathbf{x}, t))}{d t}=-\left\|\nabla\left(c \Delta n(\mathbf{x}, t)-\mu_{0}(n(\mathbf{x}, t))\right)\right\|^{2} .
$$

From this natural energy decay property of the fourth order equation $(2.5 \mathrm{a})$, it is reasonable for us to use it to approach the minimum of the total free energy, and the equilibrium state of the two-phase, single-component fluid system can be approximated by the steady solution of this equation. 


\section{Notations and Some Auxiliary Lemmas}

We investigate the numerical solution of the fourth order equation (2.5a)-(2.5b) at the time interval $\left[0, T_{m}\right]$ on the domain $\Omega=[0, L]^{2}$. Here, $T_{m}$ denotes the final time. Let $h_{1}=L / M_{1}$, $h_{2}=L / M_{2}, \triangle t=T_{m} / K, x_{i}=i h_{1}, y_{j}=j h_{2}, t_{k}=k \Delta t$. Denote

$$
\begin{aligned}
& \Omega_{h}=\left\{\left(x_{i}, y_{j}\right) \mid 0 \leq i \leq M_{1}, 0 \leq j \leq M_{2}\right\}, \quad \Omega_{\tau}=\left\{t_{k} \mid 0 \leq k \leq K\right\}, \\
& \mathcal{V}_{h}=\left\{n \mid n=\left\{n_{i j}\right\}, n_{i+M_{1}, j}=n_{i j}, n_{i, j+M_{2}}=n_{i j}\right\} .
\end{aligned}
$$

For $n \in \mathcal{V}_{h}$, denote

$$
\begin{array}{ll}
\delta_{x} n_{i+\frac{1}{2}, j}=\frac{1}{h_{1}}\left(n_{i+1, j}-n_{i, j}\right), & \delta_{y} n_{i, j+\frac{1}{2}}=\frac{1}{h_{2}}\left(n_{i, j+1}-n_{i, j}\right), \\
\delta_{x}^{2} n_{i j}=\frac{1}{h_{1}^{2}}\left(n_{i+1, j}-2 n_{i j}+n_{i-1, j}\right), & \delta_{y}^{2} n_{i j}=\frac{1}{h_{2}^{2}}\left(n_{i, j+1}-2 n_{i j}+n_{i, j-1}\right), \\
\nabla_{h} n_{i+\frac{1}{2}, j+\frac{1}{2}}=\left(\delta_{x} n_{i+\frac{1}{2}, j}, \delta_{y} n_{i, j+\frac{1}{2}}\right)^{T}, & \triangle_{h} n_{i j}=\left(\delta_{x}^{2}+\delta_{y}^{2}\right) n_{i j} .
\end{array}
$$

For a grid function $w=\left(w^{0}, w^{1}, \cdots, w^{K-1}, w^{K}\right)$ on $\Omega_{\tau}$, define

$$
\begin{array}{lll}
w^{n+\frac{1}{2}}=\frac{1}{2}\left(w^{n}+w^{n+1}\right), & \delta_{t} w^{n+\frac{1}{2}}=\frac{1}{\Delta t}\left(w^{n+1}-w^{n}\right), & 0 \leq k \leq K-1, \\
\hat{w}^{k+\frac{1}{2}}=2 w^{k-\frac{1}{2}}-w^{k-\frac{3}{2}}=w^{k}+\frac{1}{2} w^{k-1}-\frac{1}{2} w^{k-2}, & 2 \leq k \leq K-1, \\
\hat{w}^{\frac{1}{2}}=w^{0}+\frac{1}{2} w_{t}^{0} \triangle t, & \hat{w}^{\frac{3}{2}}=w^{0}+\frac{3}{2} \triangle t w_{t}^{0} . &
\end{array}
$$

For $u, v \in \mathcal{V}_{h}$, their inner product is defined as $\langle u, v\rangle=h_{1} h_{2} \sum_{i=1}^{M_{1}} \sum_{j=1}^{M_{2}} u_{i j} v_{i j}, \quad$ and their Sobolev norms as

$$
\begin{array}{rlrl}
\|u\|_{\infty} & =\max _{1 \leq i \leq M_{1}, 1 \leq j \leq M_{2}}\left|u_{i j}\right|, & \|u\|=\sqrt{\langle u, u\rangle}, \\
\left\|\delta_{x} u\right\|=\sqrt{h_{1} h_{2} \sum_{i=1}^{M_{1}} \sum_{j=1}^{M_{2}}\left|\delta_{x} u_{i-\frac{1}{2}, j}\right|^{2}}, & \left\|\delta_{y} u\right\|=\sqrt{h_{1} h_{2} \sum_{i=1}^{M_{1}} \sum_{j=1}^{M_{2}}\left|\delta_{y} u_{i, j-\frac{1}{2}}\right|^{2}}, \\
\left\|\nabla_{h} u\right\|=\sqrt{h_{1} h_{2} \sum_{i=1}^{M_{1}} \sum_{j=1}^{M_{2}}\left|\nabla_{h} u_{i+\frac{1}{2}, j+\frac{1}{2}}\right|^{2}}, & \left\|\triangle_{h} u\right\|=\sqrt{h_{1} h_{2} \sum_{i=1}^{M_{1}} \sum_{j=1}^{M_{2}}\left|\triangle_{h} u_{i j}\right|^{2}} .
\end{array}
$$

To demonstrate the solvability and the convergence of special numerical schemes, we will frequently use the following lemmas.

Lemma 3.1. ([1,2]) Let $(H,(\cdot, \cdot))$ be a finite dimensional inner product space, $\|\cdot\|$ the associated norm, and $g: H \rightarrow H$ be continuous. Assume moreover that

$$
\exists \alpha>0, \quad \forall z \in H, \quad\|z\|=\alpha, \quad\langle g(z), z\rangle \geq 0 .
$$

Then there exists an element $z^{*} \in H$, such that $g\left(z^{*}\right)=0$ and $\left\|z^{*}\right\| \leq \alpha$.

Lemma 3.2. For any grid function $u, v \in \mathcal{V}_{h}$, we have

$$
\left\langle\Delta_{h} u, v\right\rangle=\left\langle u, \Delta_{h} v\right\rangle=-\left\langle\nabla_{h} u \cdot \nabla_{h} v, 1\right\rangle .
$$


Lemma 3.3. ([15]) For any grid function $n \in \mathcal{V}_{h}$, we have

$$
\|n\|_{\infty}^{2} \leq k_{0}\|n\|\left(\left\|\triangle_{h} n\right\|+\|n\|\right)
$$

where $k_{0}$ is independent of the grid parameter $h$ and the function $n$.

Referencing to the Lemma 4.2 given by [15], we can get the following similar result.

Lemma 3.4. For any $u, v \in \mathcal{V}_{h}$ and $k \geq 1$, we have the following identity

$$
\sum_{l=0}^{k} u^{l+\frac{1}{2}} \delta_{t} v^{l+\frac{1}{2}}=\frac{1}{\Delta t}\left(u^{k+\frac{1}{2}} v^{k+1}-u^{\frac{1}{2}} v^{0}\right)-\sum_{l=1}^{k} v^{l} \frac{u^{l+\frac{1}{2}}-u^{l-\frac{1}{2}}}{\Delta t} .
$$

Proof. Observe that

$$
\begin{aligned}
& \sum_{l=0}^{k} u^{l+\frac{1}{2}} \delta_{t} v^{l+\frac{1}{2}}=\sum_{l=0}^{k} u^{l+\frac{1}{2}} \frac{v^{l+1}-v^{l}}{\Delta t} \\
= & \frac{1}{\Delta t}\left[\sum_{l=1}^{k}\left(u^{l-\frac{1}{2}}-u^{l+\frac{1}{2}}\right) v^{l}+u^{k+\frac{1}{2}} v^{k+1}-u^{\frac{1}{2}} v^{0}\right] \\
= & \frac{1}{\Delta t}\left(u^{k+\frac{1}{2}} v^{k+1}-u^{\frac{1}{2}} v^{0}\right)-\sum_{l=1}^{k} v^{l} \frac{u^{l+\frac{1}{2}}-u^{l-\frac{1}{2}}}{\Delta t} .
\end{aligned}
$$

This completes the proof.

Similarly to the approach of Lemma 4.1 provided in [15], we can obtain the following helpful lemma.

Lemma 3.5. Denote $e_{i j}^{k}=\bar{n}_{i j}^{k}-n_{i j}^{k}$, where $\bar{n}$ is the solution of (2.5a). For $n, \bar{n} \in \mathcal{V}_{h}$, there exists $\rho^{l} \in(0,1)$ and $\gamma_{1}=\rho^{l} n^{l+\frac{1}{2}}+\left(1-\rho^{l}\right) n^{l-\frac{1}{2}}, \gamma_{2}=\rho^{l} \bar{n}^{l+\frac{1}{2}}+\left(1-\rho^{l}\right) \bar{n}^{l-\frac{1}{2}}, \xi^{l} \in$ $\left(\min \left\{\gamma_{1}, \gamma_{2}\right\}, \max \left\{\gamma_{1}, \gamma_{2}\right\}\right), k, l=0,1,2, \ldots, K-1$, such that

$$
\begin{aligned}
& \frac{1}{\Delta t}\left[\mu_{0}\left(\bar{n}^{l+\frac{1}{2}}\right)-\mu_{0}\left(n^{l+\frac{1}{2}}\right)-\left(\mu_{0}\left(\bar{n}^{l-\frac{1}{2}}\right)-\mu_{0}\left(n^{l-\frac{1}{2}}\right)\right)\right] \\
= & \mu_{0}^{\prime}\left(\rho^{l} n^{l+\frac{1}{2}}+\left(1-\rho^{l}\right) n^{l-\frac{1}{2}}\right) \frac{e^{l+\frac{1}{2}}-e^{l-\frac{1}{2}}}{\Delta t}+\mu_{0}^{\prime \prime}\left(\xi^{l}\right)\left(\rho^{l} e^{l+\frac{1}{2}}+\left(1-\rho^{l}\right) e^{l-\frac{1}{2}}\right) \frac{\bar{n}^{l+\frac{1}{2}}-\bar{n}^{l-\frac{1}{2}}}{\Delta t} .
\end{aligned}
$$

Proof. Note that

$$
\begin{aligned}
& \frac{1}{\Delta t}\left[\mu_{0}\left(\bar{n}^{l+\frac{1}{2}}\right)-\mu_{0}\left(n^{l+\frac{1}{2}}\right)-\left(\mu_{0}\left(\bar{n}^{l-\frac{1}{2}}\right)-\mu_{0}\left(n^{l-\frac{1}{2}}\right)\right)\right] \\
= & \mu_{0}^{\prime}\left(\rho^{l} \bar{n}^{l+\frac{1}{2}}+\left(1-\rho^{l}\right) \bar{n}^{l-\frac{1}{2}}\right) \frac{\bar{n}^{l+\frac{1}{2}}-\bar{n}^{l-\frac{1}{2}}}{\Delta t}-\mu_{0}^{\prime}\left(\rho^{l} n^{l+\frac{1}{2}}+\left(1-\rho^{l}\right) n^{l-\frac{1}{2}}\right) \frac{n^{l+\frac{1}{2}}-n^{l-\frac{1}{2}}}{\Delta t} \\
= & {\left[\mu_{0}^{\prime}\left(\rho^{l} \bar{n}^{l+\frac{1}{2}}+\left(1-\rho^{l}\right) \bar{n}^{l-\frac{1}{2}}\right)-\mu_{0}^{\prime}\left(\rho^{l} n^{l+\frac{1}{2}}+\left(1-\rho^{l}\right) n^{l-\frac{1}{2}}\right)\right] \frac{\bar{n}^{l+\frac{1}{2}}-\bar{n}^{l-\frac{1}{2}}}{\Delta t} } \\
& \quad+\mu_{0}^{\prime}\left(\rho^{l} n^{l+\frac{1}{2}}+\left(1-\rho^{l}\right) n^{l-\frac{1}{2}}\right)\left(\frac{\bar{n}^{l+\frac{1}{2}}-\bar{n}^{l-\frac{1}{2}}}{\Delta t}-\frac{n^{l+\frac{1}{2}}-n^{l-\frac{1}{2}}}{\Delta t}\right) \\
= & \mu_{0}^{\prime}\left(\rho^{l} n^{l+\frac{1}{2}}+\left(1-\rho^{l}\right) n^{l-\frac{1}{2}}\right) \frac{e^{l+\frac{1}{2}}-e^{l-\frac{1}{2}}}{\Delta t}+\mu_{0}^{\prime \prime}\left(\xi^{l}\right)\left(\rho^{l} e^{l+\frac{1}{2}}+\left(1-\rho^{l}\right) e^{l-\frac{1}{2}}\right) \frac{\bar{n}^{l+\frac{1}{2}}-\bar{n}^{l-\frac{1}{2}}}{\Delta t} .
\end{aligned}
$$


In deriving (3.4), we treated $\mu_{0}\left(\bar{u}^{k}+\Delta t \rho \delta_{t} \bar{u}^{k+\frac{1}{2}}\right)-\mu_{0}\left(u^{k}+\Delta t \rho \delta_{t} u^{k+\frac{1}{2}}\right)$ as a function of $\rho \in[0,1]$ and then use the mean-value theorem. To derive (3.5), we have also applied the differential mid-value theorem.

\section{Crank-Nicolson Scheme}

Define the grid function $\bar{n}^{k}, \bar{f}_{0}^{k} \in \mathcal{V}_{h}$ for $0 \leq k \leq K$ on $\Omega_{h}$ as follows,

$$
\bar{n}_{i j}^{k}=n\left(x_{i}, y_{j}, t_{k}\right), \quad\left(\bar{f}_{0}\right)_{i j}^{k}=f_{0}^{k}\left(x_{i}, y_{j}\right), \quad 1 \leq i \leq M_{1}, \quad 1 \leq j \leq M_{2} .
$$

Applying Taylor expansion to (2.5a) and (2.6a) for all $1 \leq i \leq M_{1}, 1 \leq j \leq M_{2}$, we have

$$
\begin{aligned}
& \frac{\bar{n}_{i j}^{k+1}-\bar{n}_{i j}^{k}}{\triangle t}+c \triangle_{h}^{2} \bar{n}_{i j}^{k+\frac{1}{2}}-\triangle_{h} \mu_{0}\left(\bar{n}_{i j}^{k+\frac{1}{2}}\right)=R_{i j}^{k+\frac{1}{2}}, \quad 0 \leq k \leq K-1, \\
& \frac{\left(\bar{f}_{0}\right)_{i j}^{k+1}-\left(\bar{f}_{0}\right)_{i j}^{k}}{\triangle t}=\mu_{0}\left(\bar{n}^{k+\frac{1}{2}}\right) \frac{\bar{n}_{i j}^{k+1}-\bar{n}_{i j}^{k}}{\triangle t}+S_{i j}^{k+\frac{1}{2}}, \quad 0 \leq k \leq K-1,
\end{aligned}
$$

where there exists a constant $m_{1}$, such that for $0 \leq k \leq K-1$

$$
\left|R_{i j}^{k+\frac{1}{2}}\right| \leq m_{1}\left(h_{1}^{2}+h_{2}^{2}+\triangle t^{2}\right), \quad\left|S_{i j}^{k+\frac{1}{2}}\right| \leq m_{1}\left(h_{1}^{2}+h_{2}^{2}+\triangle t^{2}\right),
$$

with the initial conditions

$$
\bar{n}_{i j}^{0}=n_{0}\left(x_{i}, y_{j}\right), \quad \bar{f}_{i j}^{0}=f_{0}\left(\bar{n}_{i j}^{0}\right) .
$$

Omitting the local truncation error terms in (4.1a) and (4.1b), we can derive the Crank-Nicolson scheme of the fourth-order parabolic equation (2.5a) and (2.6a) for all $1 \leq i \leq M_{1}, 1 \leq j \leq M_{2}$, $0 \leq k \leq K-1$ as follows,

$$
\begin{aligned}
& \frac{n_{i j}^{k+1}-n_{i j}^{k}}{\triangle t}+c \triangle_{h}^{2} n_{i j}^{k+\frac{1}{2}}-\triangle_{h} \mu_{0}\left(n_{i j}^{k+\frac{1}{2}}\right)=0, \\
& \frac{\left(f_{0}\right)_{i j}^{k+1}-\left(f_{0}\right)_{i j}^{k}}{\triangle t}=\mu_{0}\left(n_{i j}^{k+\frac{1}{2}}\right) \frac{n_{i j}^{k+1}-n_{i j}^{k}}{\triangle t} .
\end{aligned}
$$

Here, the total discrete free energy at $k \Delta t, k=0,1,2, \ldots, K$, is defined as

$$
F_{h}^{k}=\frac{c}{2}\left\|\nabla_{h} n^{k}\right\|^{2}+\left\langle f_{0}^{k}, 1\right\rangle
$$

and it is updated by the equation (4.5). We note that, there is a little inconsistence between the modified version of the discrete total energy as its original one due to the fact that $f_{0}^{k} \neq f_{0}\left(n^{k}\right)$. We apply the idea proposed in the work of Qiao. et al [24], to guarantee the energy decreasing property during the evolution process. The proof of the energy stability presented below will demonstrate how it plays the role. And the improvement of the consistence between the two energy expressions will be left as one aspect of our future work.

Lemma 4.1. (Mass conservation.) The solution of the discrete equation (4.4) satisfies the mass conservation, that is, for any $0 \leq k \leq K-1$,

$$
h_{1} h_{2} \sum_{i=1}^{M_{1}} \sum_{j=1}^{M_{2}} n_{i j}^{k+1}=h_{1} h_{2} \sum_{i=1}^{M_{1}} \sum_{j=1}^{M_{2}} n_{i j}^{k} .
$$


Remark 4.1. The proof is classical. The mass conservation can be obtained spontaneously by multiplying $h_{1} h_{2} \Delta t$ to both sides of (4.4), summing for $i=1, \ldots, M_{1}, j=1, \ldots, M_{2}$, with supplement of the periodic boundary condition.

Lemma 4.2. (Energy identity.) If the total discrete free energy at $k \Delta t, k=0,1, \ldots K$, is defined by (4.6), the discrete scheme provided by (4.4)-(4.5) can guarantee the following energy identity for any time step $\Delta t>0$,

$$
\frac{F_{h}^{k+1}-F_{h}^{k}}{\Delta t}+\left\|\nabla_{h} w^{k+\frac{1}{2}}\right\|^{2}=0
$$

Proof. Define $w^{k+\frac{1}{2}}, k=0,1,2, \ldots, K-1$, as

$$
w_{i j}^{k+\frac{1}{2}}=c \triangle_{h} n_{i j}^{k+\frac{1}{2}}-\mu_{0}\left(n_{i j}^{k+\frac{1}{2}}\right) .
$$

Taking the inner product of (4.4) with $w^{k+\frac{1}{2}}$, we have

$$
\frac{1}{\Delta t}\left\langle n^{k+1}-n^{k}, c \Delta_{h} \frac{n^{k+1}+n^{k}}{2}\right\rangle-\frac{1}{\Delta t}\left\langle n^{k+1}-n^{k}, \mu_{0}\left(n^{k+\frac{1}{2}}\right)\right\rangle+\left\langle\Delta_{h} w^{k+\frac{1}{2}}, w^{k+\frac{1}{2}}\right\rangle=0 .
$$

By using integration by parts under periodic boundary condition and the equation (4.5), we have

$$
\frac{c}{2 \triangle t}\left(\left\|\nabla n^{k+1}\right\|^{2}-\left\|\nabla n^{k}\right\|^{2}\right)+\frac{1}{\triangle t}\left\langle f_{0}^{k+1}-f_{0}^{k}, 1\right\rangle+\left\|-\nabla w^{k+\frac{1}{2}}\right\|^{2}=0 .
$$

Recombining the terms in the above formula, we can get

$$
\frac{1}{\triangle t}\left(\frac{c}{2}\left\|\nabla n^{k+1}\right\|^{2}+\left\langle f_{0}^{k+1}, 1\right\rangle\right)-\frac{1}{\triangle t}\left(\frac{c}{2}\left\|\nabla n^{k}\right\|^{2}+\left\langle f_{0}^{k}, 1\right\rangle\right)+\left\|-\nabla w^{k+\frac{1}{2}}\right\|^{2}=0,
$$

which is a detailed form of (4.7). This completes the proof.

Remark 4.2. We note that, this energy stability is respect to the modified energy expression (4.6), which is a little different with the discrete form of the original energy functional (2.4). In turn, one can not obtain the $H^{1}$ bound for the numerical solution, even if the numerical solution is required to be inside the domain $\left[\theta_{0}, 1 / b-\theta_{0}\right]$, as in the following theorems.

\subsection{The unique solvability}

\subsubsection{Solvability}

Theorem 4.1. The discrete scheme (4.4)-(4.5) has at least one solution.

Proof. The scheme (4.4) for all $1 \leq i \leq M_{1}, 1 \leq j \leq M_{2}, 0 \leq k \leq K-1$, can be written as

$$
w_{i j}-n_{i j}^{k}+\frac{c \triangle t}{2} \triangle_{h}^{2} w_{i j}-\frac{\triangle t}{2} \triangle_{h} \mu_{0}\left(w_{i j}\right)=0,
$$

where $w=n^{k+\frac{1}{2}}$. Define the map

$$
g\left(w_{i j}\right)=w_{i j}-n_{i j}^{k}+\frac{c \triangle t}{2} \triangle_{h}^{2} w_{i j}-\frac{\triangle t}{2} \triangle_{h} \mu_{0}\left(w_{i j}\right) .
$$


Then

$$
\begin{aligned}
\langle g(w), w\rangle & =\|w\|^{2}-\left\langle n^{k}, w\right\rangle+\frac{c \triangle t}{2}\left\|\triangle_{h} w\right\|^{2}-\frac{\triangle t}{2}\left\langle\triangle_{h} \mu_{0}(w), w\right\rangle \\
& =\|w\|^{2}-\left\langle n^{k}, w\right\rangle+\frac{c \triangle t}{2}\left\|\triangle_{h} w\right\|^{2}+\frac{\triangle t}{2}\left\langle\mu_{0}^{\prime}(w) \nabla_{h} w \cdot \nabla_{h} w, 1\right\rangle .
\end{aligned}
$$

Therefore,

$$
\begin{aligned}
\langle g(w), w\rangle & >\left[\|w\|^{2}-\left\langle n^{k}, w\right\rangle\right]+\frac{c \triangle t}{2}\left\|\triangle_{h} w\right\|^{2}-a(T) \triangle t\left\|\nabla_{h} w\right\|^{2} \\
& \geq\left[\|w\|^{2}-\left\|n^{k}\right\|\|w\|\right]+\frac{c \triangle t}{2}\left\|\triangle_{h} w\right\|^{2}-\left(\frac{c \triangle t}{2}\left\|\triangle_{h} w\right\|^{2}+\frac{a^{2}(T) \triangle t}{2 c}\|w\|^{2}\right) \\
& =\left(1-\frac{a^{2}(T) \triangle t}{2 c}\right)\|w\|^{2}-\left\|n^{k}\right\|\|w\| \geq\left[\left(1-\frac{a^{2}(T) \triangle t}{2 c}\right)\|w\|-\left\|n^{k}\right\|\right]\|w\| .
\end{aligned}
$$

When $\triangle t<\frac{c}{a^{2}(T)}$, it follows that $\frac{a^{2}(T) \triangle t}{2 c}<\frac{1}{2}$, and

$$
(g(w), w) \geq\left(\frac{1}{2}\|w\|-\left\|n^{k}\right\|\right)\|w\|=\frac{1}{2}\left(\|w\|-2\left\|n^{k}\right\|\right)\|w\| .
$$

If $\|w\|=2\left\|n^{k}\right\|$, we have $\langle g(w), w\rangle \geq 0$. By Lemma 3.1, there is at least one solution $w$ satisfying $\|w\| \leq 2\left\|n^{k}\right\|$. The solvability of the Crank-Nicolson scheme is proved.

Remark 4.3. It has to be noted that Theorem 4.1 can only guarantee the existence of the real value of the solution $\mathbf{n}^{k+1}$ for the equation (4.4). However, the reasonable value of each component of the vector $\mathbf{n}^{k+1}$ has to be in the open subset of the set of real numbers $(0,1 / b)$. This requirement for the solution from the physical background could not be guaranteed by this theorem. Numerically, this requirement could be guaranteed by selecting appropriate temporal steps since the program can only run ahead only if all the components of the vector $\mathbf{n}^{k+1}$ are in the region $(0,1 / b)$. Furthermore, all the components of the obtained numerical solution $\mathbf{n}^{k+1}$ are in a close subset of the reasonable $(0,1 / b)$ due to the finiteness of their total number, which could be written as $\left[\theta_{0}, 1 / b-\theta_{0}\right]$, where $\theta_{0} \in(0,1 /(2 b))$ is a really small value.

In addition, the uniqueness of the reasonable solution of the discrete equation (4.4) is provided as follows.

\subsubsection{Uniqueness of the solution}

Theorem 4.2. The discrete scheme (4.4)-(4.5) has at most one solution in the region $\left[\theta_{0}, 1 / b-\theta_{0}\right]$ for any $\theta_{0} \in(0,1 /(2 b))$ if $\Delta t<2 c / M^{2}$. Here $M=\max \left\{\left|\mu^{\prime}(n)\right|: n \in\left[\theta_{0}, 1 / b-\theta_{0}\right]\right\}$.

Proof. Suppose (4.8) has another solution $z, w_{i j} \in\left[\theta_{0}, 1 / b-\theta_{0}\right]$ and $z_{i j} \in\left[\theta_{0}, 1 / b-\theta_{0}\right]$ for all $1 \leq i \leq M_{1}, 1 \leq j \leq M_{2}$, then

$$
\left(z_{i j}-n_{i j}^{k}\right)+\frac{c \triangle t}{2} \triangle_{h}^{2} z_{i j}-\frac{\triangle t}{2} \triangle_{h} \mu_{0}\left(z_{i j}\right)=0 .
$$

Let $\epsilon_{i j}=w_{i j}-z_{i j}$, subtracting (4.9) from (4.8), we have

$$
\epsilon_{i j}+\frac{c \triangle t}{2} \triangle_{h}^{2} \epsilon_{i j}-\frac{\triangle t}{2}\left[\triangle_{h} \mu_{0}\left(w_{i j}\right)-\triangle_{h} \mu_{0}\left(z_{i j}\right)\right]=0 .
$$


Taking the inner product of (4.10) with $\epsilon$, we obtain

$$
\|\epsilon\|^{2}+\frac{c \triangle t}{2}\left\|\triangle_{h} \epsilon\right\|^{2}-\frac{\triangle t}{2}\left\langle\triangle_{h} \mu_{0}(w)-\triangle_{h} \mu_{0}(z), \epsilon\right\rangle=0 .
$$

According to the Lemma 3.2, we have

$$
\frac{\triangle t}{2}\left\langle\triangle_{h} \mu_{0}(w)-\triangle_{h} \mu_{0}(z), \epsilon\right\rangle=\frac{\triangle t}{2}\left\langle\mu_{0}(w)-\mu_{0}(z), \triangle_{h} \epsilon\right\rangle .
$$

Then

$$
\begin{aligned}
\|\epsilon\|^{2}+\frac{c \triangle t}{2}\left\|\triangle_{h} \epsilon\right\|^{2} & =\frac{\triangle t}{2}\left\langle\mu_{0}(w)-\mu_{0}(z), \triangle_{h} \epsilon\right\rangle=\frac{\triangle t}{2}\left\langle\frac{\partial \mu_{0}}{\partial n}(\xi) \epsilon, \Delta_{h} \epsilon\right\rangle \\
& \leq \frac{\triangle t}{2} M\|\epsilon\|\|\Delta \epsilon\| \leq \frac{\Delta t M^{2}}{8 c}\|\epsilon\|^{2}+\frac{c \triangle t}{2}\left\|\triangle_{h} \epsilon\right\|^{2} .
\end{aligned}
$$

where $\xi=c w+(1-c) z, c \in[0,1]$, satisfies $\left|\mu^{\prime}(\xi)\right|<M$ spontaneously. Therefore,

$$
\|\epsilon\|^{2}+\frac{c \triangle t}{2}\left\|\triangle_{h} \epsilon\right\|^{2} \leq \frac{\Delta t M^{2}}{8 c}\|\epsilon\|^{2}+\frac{c \triangle t}{2}\left\|\triangle_{h} \epsilon\right\|^{2}
$$

Thus $\|\epsilon\|^{2} \leq \frac{\Delta t M^{2}}{8 c}\|\epsilon\|^{2}$. If $\Delta t<\frac{8 c}{M^{2}}, \epsilon=0$. This completes the proof.

\subsection{Convergence}

For the convenience of presentation, we first introduce the following notations.

$$
\begin{aligned}
& \alpha_{11}=\max \left\{\left|\mu_{0}^{\prime}(n)\right|: n \in\left[\epsilon_{1}, 1 / b-\epsilon_{1}\right]\right\}, \quad \alpha_{12}=\max \left\{\left|\mu_{0}^{\prime \prime}(n)\right|: n \in\left[\epsilon_{1}, 1 / b-\epsilon_{1}\right]\right\} \\
& \alpha_{13}=\frac{\alpha_{11}^{2}}{4 c}+\frac{1}{2}, \quad \alpha_{14}=\max \left\{\left|\frac{\bar{n}^{k+1}-\bar{n}^{k}}{\Delta t}\right|, k=1,2, \ldots K-1 .\right\}, \\
& \alpha_{15}=\frac{4 \alpha_{11}^{2}}{c^{2}}+\frac{2 \alpha_{12} \alpha_{14}+2 c+2}{c} \\
& \alpha_{16}=2+\frac{\alpha_{11}^{2}}{c}+\frac{4 \alpha_{11}^{2}}{c^{2}}+\frac{4 \alpha_{12} \alpha_{14}}{c}+\frac{\alpha_{11}^{2}}{c}\left(\frac{2 \alpha_{11}^{2}}{c^{2}}+\frac{\alpha_{12} \alpha_{14}}{c}\right)+\max \left\{\frac{\alpha_{12} \alpha_{14}}{c}, \frac{2 \alpha_{11}^{2}}{c^{2}}\right\}, \\
& C_{11}=\sqrt{\frac{|\Omega|}{4 \alpha_{13}}} \exp \left(2 \alpha_{13}(k+1) \Delta t\right) m_{1}, \\
& C_{12}=\sqrt[4]{\frac{\alpha_{15}}{2 \alpha_{13} \alpha_{16}} k_{0}^{2}|\Omega|^{2}} \exp \left(\left(\alpha_{13}+\frac{\alpha_{16}}{4}\right)(k+1) \Delta t\right) m_{1} .
\end{aligned}
$$

Theorem 4.3. Suppose the solution of the original fourth order equation $(2.5 a)-(2.5 b)$ is sufficiently smooth, and there exists $\epsilon_{1}$ such that for any $k=0,1, \ldots, K$, the solution of the CrankNicolson scheme (4.4), $n^{k}$ and the solution of $(4.1 a) \bar{n}^{k} \in\left[\epsilon_{1}, 1 / b-\epsilon_{1}\right]$. If $\alpha_{13} \Delta t<1 / 2$, then

$$
\left\|e^{k+1}\right\| \leq C_{11}\left(h_{1}^{2}+h_{2}^{2}+\Delta t^{2}\right)
$$

if $\alpha_{15} \Delta t<\frac{1}{2}$, we can obtain

$$
\left\|e^{k+1}\right\|_{\infty} \leq C_{12}\left(h_{1}^{2}+h_{2}^{2}+\Delta t^{2}\right)
$$


Proof. We know $\bar{n}_{i j}^{k+1}=\bar{n}\left(x_{i}, y_{j}, t_{k}\right)$ is the solution of (4.1a), and $n_{i j}^{k+1}$ is the solution of (4.4). Let $e_{i j}^{k}=\bar{n}_{i j}^{k}-n_{i j}^{k}$. Subtracting (4.4) from (4.1a), we have: for all $1 \leq i \leq M_{1}$, $1 \leq j \leq M_{2}, e_{i j}^{0}=0$, and

$$
\frac{e_{i j}^{k+1}-e_{i j}^{k}}{\triangle t}+c \triangle_{h}^{2} e_{i j}^{k+\frac{1}{2}}-\triangle_{h} \mu_{0}\left(\bar{n}_{i j}^{k+\frac{1}{2}}\right)+\triangle_{h} \mu_{0}\left(n_{i j}^{k+\frac{1}{2}}\right)=R_{i j}^{k+\frac{1}{2}}, \quad 0 \leq k \leq K-1 .
$$

Taking inner product of (4.11) with $e^{k+\frac{1}{2}}=\frac{e^{k+1}+e^{k}}{2}$, then

$$
\begin{aligned}
& \frac{\left\|e^{k+1}\right\|^{2}-\left\|e^{k}\right\|^{2}}{2 \triangle t}+c\left\|\triangle_{h} e^{k+\frac{1}{2}}\right\|^{2} \\
= & \left\langle\triangle_{h} \mu_{0}\left(\bar{n}^{k+\frac{1}{2}}\right)-\triangle_{h} \mu_{0}\left(n^{k+\frac{1}{2}}\right), e^{k+\frac{1}{2}}\right\rangle+\left\langle R^{k+\frac{1}{2}}, e^{k+\frac{1}{2}}\right\rangle .
\end{aligned}
$$

According to Lemma 3.2, we have

$$
\begin{aligned}
& \left\langle\triangle_{h} \mu_{0}\left(\bar{n}^{k+\frac{1}{2}}\right)-\triangle_{h} \mu_{0}\left(n^{k+\frac{1}{2}}\right), e^{k+\frac{1}{2}}\right\rangle \\
= & \left\langle\mu_{0}\left(\bar{n}^{k+\frac{1}{2}}\right)-\mu_{0}\left(n^{k+\frac{1}{2}}\right), \triangle_{h} e^{k+\frac{1}{2}}\right\rangle=\left\langle\mu_{0}^{\prime}\left(\xi^{k+\frac{1}{2}}\right) e^{k+\frac{1}{2}}, \Delta_{h} e^{k+\frac{1}{2}}\right\rangle \\
\leq & \alpha_{11}\left\|e^{k+\frac{1}{2}}\right\|\left\|\Delta_{h} e^{k+\frac{1}{2}}\right\| \leq \frac{\alpha_{11}^{2}}{4 c}\left\|e^{k+\frac{1}{2}}\right\|^{2}+c\left\|\triangle_{h} e^{k+\frac{1}{2}}\right\|^{2} .
\end{aligned}
$$

Using (4.2), we have

$$
\begin{gathered}
\left\langle R^{k+\frac{1}{2}}, e^{k+\frac{1}{2}}\right\rangle \leq\left\|R^{k+\frac{1}{2}}\right\|\left\|\frac{e^{k+1}+e^{k}}{2}\right\| \\
\leq \frac{|\Omega|}{2} m_{1}^{2}\left(h_{1}^{2}+h_{2}^{2}+\Delta t^{2}\right)^{2}+\frac{\left\|e^{k+1}\right\|^{2}+\left\|e^{k}\right\|^{2}}{4} .
\end{gathered}
$$

Combining all the above results, we obtain

$$
\begin{aligned}
& \left\|e^{k+1}\right\|^{2}-\left\|e^{k}\right\|^{2} \\
\leq & \left(\frac{\alpha_{11}^{2}}{4 c}+\frac{1}{2}\right)\left(\left\|e^{k+1}\right\|^{2}+\left\|e^{k}\right\|^{2}\right) \Delta t+|\Omega| m_{1}^{2}\left(h_{1}^{2}+h_{2}^{2}+\Delta t^{2}\right)^{2} \Delta t .
\end{aligned}
$$

Replacing the superscript $k$ by $l$ in (4.12) and summing up for $l$ from 0 to $k$ leads to

$$
\begin{aligned}
& \left\|e^{k+1}\right\|^{2}=\sum_{l=0}^{k}\left(\left\|e^{l+1}\right\|^{2}-\left\|e^{l}\right\|^{2}\right) \\
\leq & \sum_{l=0}^{k}\left(\frac{\alpha_{11}^{2}}{4 c}+\frac{1}{2}\right)\left(\left\|e^{l+1}\right\|^{2}+\left\|e^{l}\right\|^{2}\right) \Delta t+(k+1)|\Omega| m_{1}^{2}\left(h_{1}^{2}+h_{2}^{2}+\Delta t^{2}\right)^{2} \Delta t .
\end{aligned}
$$

Thus,

$$
\left\|e^{k+1}\right\|^{2} \leq \frac{\left(\frac{\alpha_{11}^{2}}{2 c}+1\right) \Delta t}{1-\left(\frac{\alpha_{11}^{2}}{4 c}+\frac{1}{2}\right) \Delta t} \sum_{l=0}^{k}\left\|e^{l}\right\|^{2}+\frac{(k+1)|\Omega| m_{1}^{2}}{1-\left(\frac{\alpha_{11}^{2}}{4 c}+\frac{1}{2}\right) \Delta t}\left(h_{1}^{2}+h_{2}^{2}+\Delta t^{2}\right)^{2} \Delta t .
$$

If $\Delta t \leq 1 /\left(\frac{\alpha_{11}^{2}}{2 c}+1\right)$, then $\left(\frac{\alpha_{11}^{2}}{4 c}+\frac{1}{2}\right) \Delta t \leq \frac{1}{2}$, and

$$
\left\|e^{k+1}\right\|^{2} \leq\left(\frac{\alpha_{11}^{2}}{c}+2\right) \Delta t \sum_{l=0}^{k}\left\|e^{l}\right\|^{2}+2(k+1) m_{1}^{2}\left(h_{1}^{2}+h_{2}^{2}+\Delta t^{2}\right)^{2} \Delta t .
$$


Gronwall's inequality leads to

$$
\begin{aligned}
& \left\|e^{k+1}\right\| \leq \sqrt{\frac{|\Omega|}{4 \alpha_{13}}} \exp \left(2 \alpha_{13}(k+1) \Delta t\right) m_{1}\left(h_{1}^{2}+h_{2}^{2}+\Delta t^{2}\right) \\
= & C_{11}\left(h_{1}^{2}+h_{2}^{2}+\Delta t^{2}\right) .
\end{aligned}
$$

For estimating $\left\|\Delta_{h} e^{k+1}\right\|$, we take the inner product of (4.11) with $\delta_{t} e^{k+\frac{1}{2}}=\frac{e^{k+1}-e^{k}}{\Delta t}$. With the help of Lemma 3.2, we have

$$
\begin{aligned}
& \left\|\frac{e^{k+1}-e^{k}}{\Delta t}\right\|^{2}+\frac{c}{2 \Delta t}\left(\left\|\triangle_{h} e^{k+1}\right\|^{2}-\left\|\triangle_{h} e^{k}\right\|^{2}\right) \\
= & \left\langle\mu_{0}\left(\bar{n}^{k+\frac{1}{2}}\right)-\mu_{0}\left(n^{k+\frac{1}{2}}\right), \delta_{t} \triangle_{h} e^{k+\frac{1}{2}}\right\rangle+\left\langle R^{k+\frac{1}{2}}, \frac{e^{k+1}-e^{k}}{\Delta t}\right\rangle .
\end{aligned}
$$

The last term of above formula satisfies

$$
\left\langle R^{k+\frac{1}{2}}, \frac{e^{k+1}-e^{k}}{\Delta t}\right\rangle \leq\left\|R^{k+\frac{1}{2}}\right\|\left\|\frac{e^{k+1}-e^{k}}{\Delta t}\right\| \leq \frac{|\Omega|}{2} m_{1}^{2}\left(h_{1}^{2}+h_{2}^{2}+\Delta t^{2}\right)^{2}+\frac{1}{2}\left\|\frac{e^{k+1}-e^{k}}{\Delta t}\right\|^{2} .
$$

Therefore,

$$
\begin{aligned}
& \frac{1}{2}\left\|\frac{e^{k+1}-e^{k}}{\Delta t}\right\|^{2}+\frac{c}{2 \Delta t}\left(\left\|\triangle_{h} e^{k+1}\right\|^{2}-\left\|\triangle_{h} e^{k}\right\|^{2}\right) \\
\leq & \left\langle\mu_{0}\left(\bar{n}^{k+\frac{1}{2}}\right)-\mu_{0}\left(n^{k+\frac{1}{2}}\right), \delta_{t} \triangle_{h} e^{k+\frac{1}{2}}\right\rangle+\frac{|\Omega|}{2} m_{1}^{2}\left(h_{1}^{2}+h_{2}^{2}+\Delta t^{2}\right)^{2} .
\end{aligned}
$$

Replacing the superscript $k$ by $l$ in (4.15), summing up for $l$ from 0 to $k$ and using Lemmas 3.4 and 3.5 , we obtain

$$
\begin{aligned}
& \frac{1}{2} \sum_{l=0}^{k}\left\|\frac{e^{l+1}-e^{l}}{\Delta t}\right\|^{2}+\frac{c}{2 \Delta t} \sum_{l=0}^{k}\left(\left\|\Delta_{h} e^{l+1}\right\|^{2}-\left\|\Delta_{h} e^{l}\right\|^{2}\right) \\
\leq & \sum_{l=0}^{k}\left\langle\mu_{0}\left(\bar{n}^{l+\frac{1}{2}}\right)-\mu_{0}\left(n^{l+\frac{1}{2}}\right), \delta_{t} \Delta_{h} e^{l+\frac{1}{2}}\right\rangle+\frac{|\Omega|}{2}(k+1) m_{1}^{2}\left(h_{1}^{2}+h_{2}^{2}+\Delta t^{2}\right)^{2} \\
= & \frac{1}{\Delta t}\left\langle\mu_{0}^{\prime}\left(\xi_{1}^{k+\frac{1}{2}}\right) e^{k+\frac{1}{2}}, \Delta_{h} e^{k+1}\right\rangle+\frac{|\Omega|}{2}(k+1) m_{1}^{2}\left(h_{1}^{2}+h_{2}^{2}+\Delta t^{2}\right)^{2} \\
& -\sum_{l=1}^{k}\left\langle\mu_{0}^{\prime \prime}\left(\xi_{2}^{l}\right)\left(\rho^{l} e^{l+\frac{1}{2}}+\left(1-\rho^{l}\right) e^{l-\frac{1}{2}}\right) \frac{\bar{n}^{l+\frac{1}{2}}-\bar{n}^{l-\frac{1}{2}}}{\Delta t}, \Delta_{h} e^{l}\right\rangle \\
& +\sum_{l=1}^{k}\left\langle\mu_{0}^{\prime}\left(\rho^{l} n^{l+\frac{1}{2}}+\left(1-\rho^{l}\right) n^{l-\frac{1}{2}}\right) \frac{e^{l+\frac{1}{2}}-e^{l-\frac{1}{2}}}{\Delta t}, \Delta_{h} e^{l}\right\rangle+\frac{\alpha_{12} \alpha_{14}}{2} \sum_{l=1}^{k}\left(\left\|e^{l+1}\right\|+\left\|e^{l}\right\|\right. \\
& \left.+\left\|e^{l-1}\right\|\right)\left\|\Delta_{h} e^{l}\right\|+\alpha_{11} \sum_{l=1}^{k}\left[\left\|\frac{e^{l+1}-e^{l}}{2 \Delta t}\right\|+\left\|\frac{e^{l}-e^{l-1}}{2 \Delta t}\right\|\right]\left\|\Delta_{h} e^{l}\right\| \\
\leq & \frac{c}{4 \Delta t}\left\|\Delta_{h} e^{k+1}\right\|^{2}+\frac{\alpha_{11}^{2}}{2 c \Delta t}\left(\left\|e^{k+1}\right\|^{2}+\left\|e^{k}\right\|^{2}\right)+\frac{|\Omega|}{2}(k+1) m_{1}^{2}\left(h_{1}^{2}+h_{2}^{2}+\Delta t^{2}\right)^{2} \\
& +\sum_{l=1}^{k}\left(\frac{\alpha_{12} \alpha_{14}}{4}\left\|e^{l+1}\right\|^{2}+\frac{\alpha_{12} \alpha_{14}}{4}\left\|e^{l}\right\|^{2}+\frac{\alpha_{12} \alpha_{14}}{4}\left\|e^{l-1}\right\|^{2}+\frac{3 \alpha_{12} \alpha_{14}}{4}\left\|\Delta_{h} e^{l}\right\|^{2}\right)
\end{aligned}
$$




$$
+\sum_{l=1}^{k}\left(\frac{1}{4}\left\|\frac{e^{l+1}-e^{l}}{\Delta t}\right\|^{2}+\frac{1}{4}\left\|\frac{e^{l}-e^{l-1}}{\Delta t}\right\|^{2}+\frac{\alpha_{11}^{2}}{2}\left\|\Delta_{h} e^{l}\right\|\right) .
$$

Immediately,

$$
\begin{aligned}
& \frac{c}{4 \Delta t}\left\|\Delta_{h} e^{k+1}\right\|^{2} \\
\leq & \frac{\alpha_{11}^{2}}{2 c \Delta t}\left(\left\|e^{k+1}\right\|^{2}+\left\|e^{k}\right\|^{2}\right)+\frac{|\Omega|}{2}(k+1) m_{1}^{2}\left(h_{1}^{2}+h_{2}^{2}+\Delta t^{2}\right)^{2}+\frac{\alpha_{11}^{2}}{2} \sum_{l=1}^{k}\left\|\Delta_{h} e^{l}\right\|^{2} \\
& +\sum_{l=1}^{k}\left(\frac{\alpha_{12} \alpha_{14}}{4}\left\|e^{l+1}\right\|^{2}+\frac{\alpha_{12} \alpha_{14}}{4}\left\|e^{l}\right\|^{2}+\frac{\alpha_{12} \alpha_{14}}{4}\left\|e^{l-1}\right\|^{2}+\frac{3 \alpha_{12} \alpha_{14}}{4}\left\|\Delta_{h} e^{l}\right\|^{2}\right) .
\end{aligned}
$$

It naturally gives

$$
\begin{aligned}
& \left\|\Delta_{h} e^{k+1}\right\|^{2} \\
\leq & \frac{2 \alpha_{11}^{2}}{c^{2}}\left(\left\|e^{k+1}\right\|^{2}+\left\|e^{k}\right\|^{2}\right)+\frac{2|\Omega|}{c}(k+1) m_{1}^{2}\left(h_{1}^{2}+h_{2}^{2}+\Delta t^{2}\right)^{2} \Delta t \\
& +\sum_{l=1}^{k}\left[\frac{\alpha_{12} \alpha_{14}}{c}\left(\left\|e^{l+1}\right\|^{2}+\left\|e^{l}\right\|^{2}+\left\|e^{l-1}\right\|^{2}\right)+\left(\frac{3 \alpha_{12} \alpha_{14}}{c}+\frac{2 \alpha_{11}^{2}}{c}\right)\left\|\Delta_{h} e^{l}\right\|^{2}\right] \Delta t .
\end{aligned}
$$

Combining (4.13) and (4.17), we obtain

$$
\begin{aligned}
& \quad\left\|\Delta_{h} e^{k+1}\right\|^{2}+\left\|e^{k+1}\right\|^{2} \\
& \leq\left(1+\frac{2 \alpha_{11}^{2}}{c^{2}}+\frac{\alpha_{12} \alpha_{14}}{c}\right)\left\|e^{k+1}\right\|^{2}+\max \left\{\frac{3 \alpha_{12} \alpha_{14}}{c}, \frac{2 \alpha_{12} \alpha_{14}}{c}+\frac{2 \alpha_{11}^{2}}{c^{2}}\right\} \Delta t \sum_{l=1}^{k}\left\|e^{l}\right\|^{2} \\
& \quad+\left(\frac{3 \alpha_{12} \alpha_{14}}{c}+\frac{2 \alpha_{11}^{2}}{c}\right) \sum_{l=1}^{k}\left\|\Delta_{h} e^{l}\right\|^{2} \Delta t+\frac{2|\Omega|}{c}(k+1) m_{1}^{2}\left(h_{1}^{2}+h_{2}^{2}+\Delta t^{2}\right)^{2} \Delta t \\
& \leq\left[2+\frac{\alpha_{11}^{2}}{c}+\frac{4 \alpha_{11}^{2}}{c^{2}}+\frac{4 \alpha_{12} \alpha_{14}}{c}+\frac{\alpha_{11}^{2}}{c}\left(\frac{2 \alpha_{11}^{2}}{c^{2}}+\frac{\alpha_{12} \alpha_{14}}{c}\right)\right. \\
& \left.\quad+\max \left\{\frac{\alpha_{12} \alpha_{14}}{c}, \frac{2 \alpha_{11}^{2}}{c^{2}}\right\}\right] \Delta t \sum_{l=0}^{k}\left\|e^{l}\right\|^{2}+\left(\frac{4 \alpha_{11}^{2}}{c^{2}}+\frac{2 \alpha_{12} \alpha_{14}+2 c+2}{c}\right)(k \\
& \quad+1)|\Omega| m_{1}^{2}\left(h_{1}^{2}+h_{2}^{2}+\Delta t^{2}\right)^{2} \Delta t+\frac{3 \alpha_{12} \alpha_{14}+2 \alpha_{11}^{2}}{c} \sum_{l=1}^{k}\left\|\Delta_{h} e^{l}\right\|^{2} \Delta t \\
& \leq \alpha_{16} \Delta t \sum_{l=1}^{k}\left(\left\|e^{l}\right\|^{2}+\left\|\Delta_{h} e^{l}\right\|^{2}\right)+(k+1) \alpha_{15}|\Omega| m_{1}^{2}\left(h_{1}^{2}+h_{2}^{2}+\Delta t^{2}\right)^{2} \Delta t .
\end{aligned}
$$

Note that, the derivation of the last step bases on the fact that $\alpha_{11}^{2} / c<\alpha_{11}^{2} / c^{2}$ due to the coefficient $c \in(0,1)$. Gronwall's inequality yields

$$
\left\|e^{k+1}\right\|^{2}+\left\|\Delta_{h} e^{k+1}\right\|^{2} \leq \frac{\alpha_{15}}{\alpha_{16}} \exp \left(\alpha_{16}(k+1) \Delta t\right) \cdot|\Omega|\left[m_{1}\left(h_{1}^{2}+h_{2}^{2}+\Delta t^{2}\right)\right]^{2},
$$

where $k=0,1, \ldots, K-1$. Using Lemma 3.3 and (4.14), we can get

$$
\left\|e^{k+1}\right\|_{\infty}^{2} \leq k_{0}\left\|e^{k+1}\right\|\left(\left\|\triangle_{h} e^{k+1}\right\|+\left\|e^{k+1}\right\|\right)
$$




$$
\begin{aligned}
& \leq k_{0} \sqrt{2\left\|e^{k+1}\right\|^{2}\left(\left\|e^{k+1}\right\|^{2}+\left\|\triangle_{h} e^{k+1}\right\|^{2}\right)} \\
& \leq k_{0} \sqrt{\frac{\alpha_{15}}{2 \alpha_{13} \alpha_{16}}} \exp \left(\left(2 \alpha_{13}+\frac{\alpha_{16}}{2}\right)(k+1) \Delta t\right) m_{1}^{2}|\Omega|\left(h_{1}^{2}+h_{2}^{2}+\Delta t^{2}\right)^{2} .
\end{aligned}
$$

Therefore,

$$
\left\|e^{k+1}\right\|_{\infty} \leq C_{12}\left(h_{1}^{2}+h_{2}^{2}+\triangle t^{2}\right) .
$$

The completes the proof of the theorem.

\section{A Second Order Linearized Scheme}

Applying Taylor expansion to (2.5a), (2.6a) for all $1 \leq i \leq M_{1}, 1 \leq j \leq M_{2}$, we have

$$
\begin{array}{ll}
\frac{\bar{n}_{i j}^{k+1}-\bar{n}_{i j}^{k}}{\triangle t}+c \triangle_{h}^{2} \bar{n}_{i j}^{k+\frac{1}{2}}-\triangle_{h} \mu_{0}\left(\hat{\bar{n}}_{i j}^{k+\frac{1}{2}}\right)=\hat{R}_{i j}^{k+\frac{1}{2}}, & 0 \leq k \leq K-1, \\
\frac{\left(\bar{f}_{0}\right)_{i j}^{k+1}-\left(\bar{f}_{0}\right)_{i j}^{k}}{\triangle t}=\mu_{0}\left(\hat{\bar{n}}_{i j}^{k+\frac{1}{2}}\right) \frac{\bar{n}_{i j}^{k+1}-\bar{n}_{i j}^{k}}{\triangle t}+\hat{S}_{i j}^{k+\frac{1}{2}}, & 0 \leq k \leq K-1,
\end{array}
$$

with the following initial conditions

$$
\bar{n}\left(\mathbf{x}_{i j}, 0\right)=n_{0}\left(\mathbf{x}_{i j}\right), \quad \bar{f}_{0}\left(n\left(\mathbf{x}_{i j}, 0\right)\right)=f_{0}^{0}\left(n\left(\mathbf{x}_{i j}\right)\right),
$$

where there exists a constant $m_{2}$ such that

$$
\left|\hat{R}_{i j}^{k+\frac{1}{2}}\right| \leq m_{2}\left(h_{1}^{2}+h_{2}^{2}+\triangle t^{2}\right), \quad\left|\hat{S}_{i j}^{k+\frac{1}{2}}\right| \leq m_{2}\left(h_{1}^{2}+h_{2}^{2}+\triangle t^{2}\right), \quad 0 \leq k \leq K-1 .
$$

The difference scheme is constructed by omitting the local truncation error terms in the above two equations as follows: for all $1 \leq i \leq M_{1}, 1 \leq j \leq M_{2}$,

$$
\frac{n_{i j}^{k+1}-n_{i j}^{k}}{\triangle t}+c \triangle_{h}^{2} n_{i j}^{k+\frac{1}{2}}-\triangle_{h} \mu_{0}\left(\hat{n}_{i j}^{k+\frac{1}{2}}\right)=0, \quad 0 \leq k \leq K-1,
$$

subject to the initial condition

$$
n\left(\mathbf{x}_{i j}, 0\right)=n_{0}\left(\mathbf{x}_{i j}\right) .
$$

As for all $1 \leq i \leq M_{1}, 1 \leq j \leq M_{2}$, the discrete energy density $\left(f_{0}\right)_{i j}^{k+1}$ is computed by [24]

$$
\frac{\left(f_{0}\right)_{i j}^{k+1}-\left(f_{0}\right)_{i j}^{k}}{\triangle t}=\mu_{0}\left(\hat{n}_{i j}^{k+\frac{1}{2}}\right) \frac{n_{i j}^{k+1}-n_{i j}^{k}}{\triangle t}, \quad 0 \leq k \leq K-1,
$$

with initial value

$$
f_{0}\left(n\left(\mathbf{x}_{i j}, 0\right)\right)=f_{0}\left(n_{0}\left(\mathbf{x}_{i j}\right)\right) .
$$

Similarly to derivations of the mass conservation and the energy decay property of the CrankNicolson scheme, these properties of the second order linearized scheme could be given by the following two lemmas. 
Lemma 5.1. (Mass conservation.) The solution of the discrete equation (5.4a)-(5.4b) also satisfies the mass conservation if periodic boundary condition is given, which means that for any $0 \leq k \leq K-1$,

$$
h_{1} h_{2} \sum_{i=1}^{M_{1}} \sum_{j=1}^{M_{2}} n_{i j}^{k+1}=h_{1} h_{2} \sum_{i=1}^{M_{1}} \sum_{j=1}^{M_{2}} n_{i j}^{k}
$$

Lemma 5.2. (Energy identity.) If the discrete total energy at $k \Delta t, k=0,1, \ldots K$, is defined by (4.6), then the discrete scheme provided by (5.4a)-(5.5a) also can guarantee the energy identity for any time step $\Delta t>0$ as follows,

$$
\frac{F_{h}^{k+1}-F_{h}^{k}}{\triangle t}+\left\|-\nabla_{h} \hat{w}^{k+\frac{1}{2}}\right\|^{2}=0
$$

where $\hat{w}^{k+\frac{1}{2}}=c \triangle_{h} n^{k+\frac{1}{2}}-\mu_{0}\left(\hat{n}^{k+\frac{1}{2}}\right)$.

\subsection{The unique solvability and the convergence of the linearized scheme}

Theorem 5.1. The linearized scheme (5.4a)-(5.4b) is uniquely solvable.

Proof. The scheme (5.4a) for all $1 \leq i \leq M_{1}, 1 \leq j \leq M_{2}, 0 \leq k \leq K-1$ can be written as

$$
n_{i j}^{k+1}+\frac{c \triangle t}{2} \triangle_{h}^{2} n_{i j}^{k+1}=n_{i j}^{k}-\frac{c \triangle t}{2} \triangle_{h}^{2} n_{i j}^{k}+\Delta t \triangle_{h} \mu_{0}\left(\hat{n}_{i j}^{k+\frac{1}{2}}\right) .
$$

Suppose $n^{k}, \hat{n}^{k+\frac{1}{2}}$ have been determined. Then (5.7) is a linear equation about $n^{k+1}$. Consider its homogenous system as follows,

$$
n_{i j}^{k+1}+\frac{c \triangle t}{2} \triangle_{h}^{2} n_{i j}^{k+1}=0, \quad 1 \leq i \leq M_{1}, \quad 1 \leq j \leq M_{2} .
$$

Taking inner product of (5.8) with $n^{k+1}$, we have

$$
\left\|n^{k+1}\right\|^{2}+\frac{c \triangle t}{2}\left\|\triangle_{h} n^{k+1}\right\|^{2}=0 .
$$

It requires $n^{k+1}=0$. Therefore (5.7) has a unique solution.

Remark 5.1. Also, the above theorem can not guarantee that each component of the vector $\mathbf{n}^{k+1}$ is in the open subset of the set of real numbers $(0,1 / b)$. So far, we can only guarantee this from our numerical implementations. The exploration of theoretical analysis of this kind of discrete or continuous partial differential equations with high nonlinearity and singularity needs further future efforts.

For the convenience of presentation, the following notations are introduced.

$$
\begin{aligned}
& \alpha_{21}=\max \left\{\left|\mu_{0}^{\prime}(n)\right|: n \in\left[\frac{\hat{\theta}}{2}, \frac{1}{b}-\frac{\hat{\theta}}{2}\right]\right\}, \quad \alpha_{22}=\max \left\{\left|\mu_{0}^{\prime \prime}(n)\right|: n \in\left[\frac{\hat{\theta}}{2}, \frac{1}{b}-\frac{\hat{\theta}}{2}\right]\right\}, \\
& \alpha_{23}=\frac{2 \alpha_{21}^{2}}{c}+1, \quad \alpha_{24}=\max \left\{\left|\frac{\bar{n}^{k+1}-\bar{n}^{k}}{\Delta t}\right|, k=0,1, \ldots K-1 .\right\},
\end{aligned}
$$




$$
\begin{aligned}
& \alpha_{25}=\frac{16 \alpha_{21}^{2}+1}{c}+1, \quad \alpha_{26}=\max \left\{\frac{13 \alpha_{23}^{2}}{4}, 2 \alpha_{21}^{2}+4 \alpha_{22} \alpha_{24}\right\}, \\
& \alpha_{27}=\max \left\{2 \alpha_{23}+\frac{16 \alpha_{23} \alpha_{21}^{2}}{c^{2}}+\frac{5 \alpha_{22} \alpha_{24}}{c}, \frac{2 \alpha_{26}}{c}\right\}, \\
& C_{21}=\exp \left(\alpha_{23} t\right) \sqrt{\frac{|\Omega|}{\alpha_{23}}} m_{2}, \quad C_{22}=\sqrt[4]{\frac{2 \alpha_{25}}{\alpha_{23} \alpha_{27}} k_{0}^{2}|\Omega|^{2}} \exp \left(\left(\frac{\alpha_{23}+\alpha_{27}}{2}\right) T_{m}\right) m_{2} .
\end{aligned}
$$

Theorem 5.2. Suppose the solution $\bar{n}(\mathbf{x}, t)$ to the fourth order equation $(2.5 a)-(2.5 b)$ is sufficiently smooth, and there is a $\hat{\theta} \in(0,1 /(2 b))$, such that $\bar{n}_{i j}^{k} \in[\hat{\theta}, 1 / b-\hat{\theta}]$ for all $1 \leq i \leq M_{1}$, $1 \leq j \leq M_{2}, k=0,1, . . K$. If

$$
C_{22}\left(h_{1}^{2}+h_{2}^{2}+\Delta t^{2}\right) \leq \frac{1}{2} \hat{\theta}
$$

then the difference scheme (5.4a)-(5.4b) is convergent with second order in both time and space in the following detailed form

$$
\left\|e^{k+1}\right\| \leq C_{21}\left(h_{1}^{2}+h_{2}^{2}+\Delta t^{2}\right), \quad\left\|e^{k+1}\right\|_{\infty} \leq C_{22}\left(h_{1}^{2}+h_{2}^{2}+\Delta t^{2}\right) .
$$

Proof. Since

$$
\hat{\bar{n}}^{\frac{1}{2}}=\bar{n}^{0}+\frac{\Delta t}{2} \bar{n}_{t}^{0}=n^{0}+\frac{\Delta t}{2} n_{t}^{0}=\hat{n}^{\frac{1}{2}}, \quad \hat{\bar{n}}^{\frac{3}{2}}=\bar{n}^{0}+\frac{3 \Delta t}{2} \bar{n}_{t}^{0}=n^{0}+\frac{3 \Delta t}{2} n_{t}^{0}=\hat{n}^{\frac{3}{2}},
$$

we have

$$
\mu_{0}\left(\hat{\bar{n}}^{\frac{1}{2}}\right)-\mu_{0}\left(\hat{n}^{\frac{1}{2}}\right)=0, \quad \mu_{0}\left(\hat{\bar{n}}^{\frac{3}{2}}\right)-\mu_{0}\left(\hat{n}^{\frac{3}{2}}\right)=0 .
$$

Subtracting (5.4a) from (5.1a) for all $1 \leq i \leq M_{1}, 1 \leq j \leq M_{2}$, we have $e_{i j}^{0}=0$ and

$$
\begin{aligned}
& \frac{e_{i j}^{k+1}-e_{i j}^{k}}{\triangle t}+c \triangle_{h}^{2} e_{i j}^{k+\frac{1}{2}}=\hat{R}_{i j}^{k+\frac{1}{2}}, \quad k=0,1, \\
& \frac{e_{i j}^{k+1}-e_{i j}^{k}}{\triangle t}+c \triangle_{h}^{2} e_{i j}^{k+\frac{1}{2}}-\triangle_{h} \mu_{0}\left(\hat{\bar{n}}_{i j}^{k+\frac{1}{2}}\right)+\triangle_{h} \mu_{0}\left(\hat{n}_{i j}^{k+\frac{1}{2}}\right)=\hat{R}_{i j}^{k+\frac{1}{2}}, \quad 2 \leq k \leq K-1 .
\end{aligned}
$$

For the first step, taking inner product of (5.12) at $k=0$ with $e^{\frac{1}{2}}=\frac{e^{0}+e^{1}}{2}$ yields

$$
\frac{\left\|e^{1}\right\|^{2}-\left\|e^{0}\right\|^{2}}{2 \triangle t}+c\left\|\triangle_{h}\left(\frac{e^{0}+e^{1}}{2}\right)\right\|^{2}=\left\langle\hat{R}^{\frac{1}{2}}, \frac{e^{0}+e^{1}}{2}\right\rangle .
$$

For the initial value, $\left\|e^{0}\right\|=0$, so

$$
\frac{\left\|e^{1}\right\|^{2}}{2 \triangle t}+c\left\|\triangle_{h} \frac{e^{1}}{2}\right\|^{2}=\left\langle\hat{R}^{\frac{1}{2}}, \frac{e^{1}}{2}\right\rangle .
$$

Using (5.3), we get

$$
\frac{\left\|e^{1}\right\|^{2}}{2 \triangle t}+\frac{c}{4}\left\|\triangle_{h} e^{1}\right\|^{2} \leq \frac{\left\|e^{1}\right\|^{2}}{4 \triangle t}+\frac{\triangle t|\Omega| m_{2}^{2}\left(h_{1}^{2}+h_{2}^{2}+\triangle t^{2}\right)^{2}}{4},
$$

It yields

$$
\left\|e^{1}\right\| \leq \sqrt{|\Omega|} m_{2} \Delta t\left(h_{1}^{2}+h_{2}^{2}+\triangle t^{2}\right)
$$




$$
\left\|\triangle_{h} e^{1}\right\| \leq \sqrt{\frac{|\Omega| \triangle t}{c}} m_{2}\left(h_{1}^{2}+h_{2}^{2}+\triangle t^{2}\right) .
$$

According to Lemma 3.3,

$$
\begin{aligned}
\left\|e^{1}\right\|_{\infty}^{2} & \leq k_{0}\left(\left\|\triangle_{h} e^{1}\right\|\left\|e^{1}\right\|+\left\|e^{1}\right\|^{2}\right) \\
& \leq k_{0}\left[\sqrt{\frac{\triangle t}{c}} m_{2}^{2}|\Omega| \triangle t\left(h_{1}^{2}+h_{2}^{2}+\triangle t^{2}\right)^{2}+|\Omega| m_{2}^{2} \triangle t^{2}\left(h_{1}^{2}+h_{2}^{2}+\triangle t^{2}\right)^{2}\right]
\end{aligned}
$$

If $\Delta t<1 / c$, then

$$
\left\|e^{1}\right\|_{\infty}^{2} \leq \frac{2|\Omega| k_{0} \Delta t}{c} m_{2}^{2}\left(h_{1}^{2}+h_{2}^{2}+\Delta t^{2}\right)^{2}
$$

Besides, from (5.14) we also obtain

$$
\frac{\left\|e^{1}\right\|^{2}}{2 \triangle t}+\frac{c}{4}\left\|\triangle_{h} e^{1}\right\|^{2} \leq \frac{\left\|e^{1}\right\|^{2}}{4}+\frac{|\Omega| m_{2}^{2}\left(h_{1}^{2}+h_{2}^{2}+\Delta t^{2}\right)^{2}}{4} .
$$

Therefore,

$$
\frac{\left\|e^{1}\right\|^{2}}{2 \triangle t} \leq \frac{\left\|e^{1}\right\|^{2}}{4}+\frac{|\Omega| m_{2}^{2}\left(h_{1}^{2}+h_{2}^{2}+\Delta t^{2}\right)^{2}}{4} .
$$

Secondly, to get the error at second time step $\left\|e^{2}\right\|_{\infty}$, we take the inner product of (5.12) with $k=1$ and $e^{\frac{3}{2}}=\frac{e^{1}+e^{2}}{2}$

$$
\frac{\left\|e^{2}\right\|^{2}-\left\|e^{1}\right\|^{2}}{2 \triangle t}+c\left\|\triangle_{h}\left(\frac{e^{1}+e^{2}}{2}\right)\right\|^{2}=\left\langle\hat{R}^{\frac{3}{2}}, \frac{e^{1}+e^{2}}{2}\right\rangle .
$$

Applying (5.3), we have

$$
\frac{\left\|e^{2}\right\|^{2}-\left\|e^{1}\right\|^{2}}{2 \triangle t} \leq \frac{\left\|e^{1}\right\|^{2}+\left\|e^{2}\right\|^{2}}{4 \triangle t}+\frac{|\Omega| \triangle t}{2} m_{2}^{2}\left(h_{1}^{2}+h_{2}^{2}+\triangle t^{2}\right)^{2} .
$$

From (5.19) and the estimation of $\left\|e^{1}\right\|^{2}$ by (5.16), we can get

$$
\left\|e^{2}\right\| \leq \sqrt{5|\Omega|} m_{2} \triangle t\left(h_{1}^{2}+h_{2}^{2}+\triangle t^{2}\right) .
$$

To get the estimation of $\left\|\Delta e^{2}\right\|$, taking the inner product of (5.12) when $k=1$ with $\frac{e^{2}-e^{1}}{\Delta t}$, we have

$$
\begin{aligned}
& \left\|\frac{e^{2}-e^{1}}{\triangle t}\right\|^{2}+\frac{c}{2 \Delta t}\left(\left\|\triangle_{h} e^{2}\right\|^{2}-\left\|\triangle_{h} e^{1}\right\|^{2}\right) \\
= & \left\langle\hat{R}^{\frac{3}{2}}, \frac{e^{2}-e^{1}}{\Delta t}\right\rangle \leq\left\|\frac{e^{2}-e^{1}}{\triangle t}\right\|^{2}+\frac{|\Omega|}{4} m_{2}^{2}\left(h_{1}^{2}+h_{2}^{2}+\triangle t^{2}\right)^{2},
\end{aligned}
$$

from which, we obtain

$$
\left\|\triangle_{h} e^{2}\right\|^{2} \leq\left\|\triangle_{h} e^{1}\right\|^{2}+\frac{|\Omega| \Delta t}{2 c} m_{2}^{2}\left(h_{1}^{2}+h_{2}^{2}+\triangle t^{2}\right)^{2} .
$$


Combining the above equation with (5.17), we get

$$
\left\|\triangle_{h} e^{2}\right\|^{2} \leq \frac{3|\Omega| \Delta t}{2 c} m_{2}^{2}\left(h_{1}^{2}+h_{2}^{2}+\Delta t^{2}\right)^{2} .
$$

Similar to the first case, combining the equation (5.20) and (5.21) leads to

$$
\begin{aligned}
& \left\|e^{2}\right\|_{\infty}^{2} \leq k_{0}\left(\left\|\triangle_{h} e^{2}\right\|\left\|e^{2}\right\|+\left\|e^{2}\right\|^{2}\right) \\
\leq & k_{0}\left[\sqrt{\frac{15 \Delta t}{2 c}}|\Omega| m_{2}^{2} \triangle t\left(h_{1}^{2}+h_{2}^{2}+\triangle t^{2}\right)^{2}+5|\Omega| m_{2}^{2} \triangle t^{2}\left(h_{1}^{2}+h_{2}^{2}+\triangle t^{2}\right)^{2}\right] .
\end{aligned}
$$

If $\Delta t<1 / c$, then

$$
\left\|e^{2}\right\|_{\infty}^{2} \leq \frac{8 k_{0}|\Omega| \triangle t}{c} m_{2}^{2}\left(h_{1}^{2}+h_{2}^{2}+\triangle t^{2}\right)^{2}
$$

At the third step, we derive the estimation for $\left\|e^{k+1}\right\|$ and $\left\|e^{k+1}\right\|_{\infty}$ by mathematical induction. Suppose (5.11) is true for $l$ from 0 to $k(0 \leq k \leq K-1)$. Then if (5.10) is satisfied, we have

$$
\left\|e^{k}\right\|_{\infty} \leq C_{22}\left(h_{1}^{2}+h_{2}^{2}+\Delta t^{2}\right) \leq \frac{1}{2} \hat{\theta}, \quad 1 \leq l \leq k
$$

Then it follows that

$$
n_{i j}^{k} \in[\hat{\theta} / 2,1 / b-\hat{\theta} / 2], \quad 1 \leq i \leq M_{1}, \quad 1 \leq j \leq M_{2}, \quad 0 \leq l \leq k
$$

Taking inner product of (5.13) with $e^{k+\frac{1}{2}}=\frac{e^{k}+e^{k+1}}{2}$, we obtain

$$
\begin{aligned}
& \frac{\left\|e^{k+1}\right\|^{2}-\left\|e^{k}\right\|^{2}}{2 \triangle t}+c\left\|\triangle_{h} e^{k+\frac{1}{2}}\right\|^{2} \\
= & \left\langle\triangle_{h} \mu_{0}\left(\hat{\bar{n}}^{k+\frac{1}{2}}\right)-\triangle_{h} \mu_{0}\left(\hat{n}^{k+\frac{1}{2}}\right), e^{k+\frac{1}{2}}\right\rangle+\left\langle\hat{R}^{k+\frac{1}{2}}, \frac{e^{k}+e^{k+1}}{2}\right\rangle .
\end{aligned}
$$

Since

$$
\begin{aligned}
& \hat{\bar{n}}^{k+\frac{1}{2}}-\hat{n}^{k+\frac{1}{2}} \\
= & \bar{n}^{k}+\frac{1}{2} \bar{n}^{k-1}-\frac{1}{2} \bar{n}^{k-2}-\left(n^{k}+\frac{1}{2} n^{k-1}-\frac{1}{2} n^{k-2}\right)=e^{k}+\frac{1}{2} e^{k-1}-\frac{1}{2} e^{k-2},
\end{aligned}
$$

we have

$$
\mu_{0}\left(\hat{\bar{n}}^{k+\frac{1}{2}}\right)-\mu_{0}\left(\hat{n}^{k+\frac{1}{2}}\right)=\mu^{\prime}\left(\hat{\xi}^{k+\frac{1}{2}}\right)\left(e^{k}+\frac{1}{2} e^{k-1}-\frac{1}{2} e^{k-2}\right),
$$

where $\hat{\xi}^{k+\frac{1}{2}}=\hat{\lambda}^{k+\frac{1}{2}} \hat{\bar{n}}^{k+\frac{1}{2}}+\left(1-\hat{\lambda}^{k+\frac{1}{2}}\right) \hat{n}^{k+\frac{1}{2}}, \hat{\lambda}^{k+\frac{1}{2}} \in[0,1]$. According to Lemma 3.2, we get

$$
\begin{aligned}
& \left\langle\triangle_{h} \mu_{0}\left(\hat{\bar{n}}^{k+\frac{1}{2}}\right)-\triangle_{h} \mu_{0}\left(\hat{n}^{k+\frac{1}{2}}\right), e^{k+\frac{1}{2}}\right\rangle=\left\langle\mu_{0}\left(\hat{\bar{n}}^{k+\frac{1}{2}}\right)-\mu_{0}\left(\hat{n}^{k+\frac{1}{2}}\right), \triangle_{h} e^{k+\frac{1}{2}}\right\rangle \\
\leq & \left\|\mu^{\prime}\left(\hat{\xi}^{k+\frac{1}{2}}\right)\left(e^{k}+\frac{1}{2} e^{k-1}-\frac{1}{2} e^{k-2}\right)\right\|\left\|\triangle_{h} e^{k+\frac{1}{2}}\right\| \\
\leq & \alpha_{21}\left[\left\|e^{k}\right\|+\frac{1}{2}\left\|e^{k-1}\right\|+\frac{1}{2}\left\|e^{k-2}\right\|\right]\left\|\triangle_{h} e^{k+\frac{1}{2}}\right\|
\end{aligned}
$$




$$
\leq \frac{\alpha_{21}^{2}}{2 c}\left[\left\|e^{k}\right\|^{2}+\frac{1}{2}\left\|e^{k-1}\right\|^{2}+\frac{1}{2}\left\|e^{k-2}\right\|^{2}\right]+c\left\|\Delta_{h} e^{k+\frac{1}{2}}\right\|^{2} .
$$

Whereupon

$$
\begin{aligned}
& \frac{\left\|e^{k+1}\right\|^{2}-\left\|e^{k}\right\|^{2}}{2 \triangle t}+c\left\|\triangle_{h} e^{k+\frac{1}{2}}\right\|^{2} \\
\leq & \frac{\alpha_{21}^{2}}{2 c}\left[\left\|e^{k}\right\|^{2}+\frac{1}{2}\left\|e^{k-1}\right\|^{2}+\frac{1}{2}\left\|e^{k-2}\right\|^{2}\right]+c\left\|\Delta_{h} e^{k+\frac{1}{2}}\right\|^{2}+\left\langle\hat{R}^{k+\frac{1}{2}}, \frac{e^{k}+e^{k+1}}{2}\right\rangle .
\end{aligned}
$$

Accordingly,

$$
\begin{aligned}
& \frac{\left\|e^{k+1}\right\|^{2}-\left\|e^{k}\right\|^{2}}{2 \triangle t} \\
\leq & \frac{\alpha_{21}^{2}}{2 c}\left[\left\|e^{k}\right\|^{2}+\frac{1}{2}\left\|e^{k-1}\right\|^{2}+\frac{1}{2}\left\|e^{k-2}\right\|^{2}\right]+\frac{\left\|e^{k}\right\|^{2}+\left\|e^{k+1}\right\|^{2}}{4}+\frac{|\Omega|}{2} m_{2}^{2}\left(h_{1}^{2}+h_{2}^{2}+\triangle t^{2}\right)^{2} .
\end{aligned}
$$

Replacing the superscript $k$ by $l$ in (5.25) and summing up for $l$ from 2 to $k$, we get

$$
\begin{aligned}
\sum_{l=2}^{k} \frac{\left\|e^{l+1}\right\|^{2}-\left\|e^{l}\right\|^{2}}{2 \triangle t} \leq & \frac{\alpha_{21}^{2}}{2 c}\left[\sum_{l=2}^{k}\left\|e^{l}\right\|^{2}+\frac{1}{2} \sum_{l=1}^{k-1}\left\|e^{l}\right\|^{2}+\frac{1}{2} \sum_{l=1}^{k-2}\left\|e^{l}\right\|^{2}\right] \\
& +\sum_{l=2}^{k} \frac{\left\|e^{l}\right\|^{2}+\left\|e^{l+1}\right\|^{2}}{4}+\frac{|\Omega|}{2} \sum_{l=2}^{k} m_{2}^{2}\left(h_{1}^{2}+h_{2}^{2}+\triangle t^{2}\right)^{2}
\end{aligned}
$$

Combining (5.18) with (5.26) and multiplying $2 \Delta t$ to both side, we obtain

$$
\begin{aligned}
& \left(1-\frac{\Delta t}{2}\right)\left\|e^{k+1}\right\|^{2} \\
\leq & \frac{\alpha_{21}^{2}}{c}\left[\sum_{l=2}^{k}\left\|e^{l}\right\|^{2}+\frac{1}{2} \sum_{l=1}^{k-1}\left\|e^{l}\right\|^{2}+\frac{1}{2} \sum_{l=1}^{k-2}\left\|e^{l}\right\|^{2}\right] \Delta t+\frac{\Delta t}{2}\left[\sum_{l=2}^{k}\left\|e^{l}\right\|^{2}+\sum_{l=3}^{k}\left\|e^{l}\right\|^{2}\right] \\
& +|\Omega| \Delta t \sum_{l=2}^{k} m_{2}^{2}\left(h_{1}^{2}+h_{2}^{2}+\Delta t^{2}\right)^{2}+\frac{\left\|e^{2}\right\|^{2}+\left\|e^{1}\right\|^{2}}{2} \Delta t \\
& +|\Omega| \Delta t m_{2}^{2}\left(h_{1}^{2}+h_{2}^{2}+\Delta t^{2}\right)^{2}+\frac{\left\|e^{1}\right\|^{2} \Delta t}{2}+\frac{|\Omega| m_{2}^{2}\left(h_{1}^{2}+h_{2}^{2}+\Delta t^{2}\right)^{2}}{2} \Delta t \\
\leq & \frac{2 \alpha_{21}^{2}}{c} \Delta t \sum_{l=1}^{k}\left\|e^{l}\right\|^{2}+\Delta t \sum_{l=1}^{k}\left\|e^{l}\right\|^{2}+(k+1)|\Omega| \Delta t m_{2}^{2}\left(h_{1}^{2}+h_{2}^{2}+\Delta t^{2}\right)^{2} \\
= & \alpha_{23} \Delta t \sum_{l=1}^{k}\left\|e^{l}\right\|^{2}+(k+1)|\Omega| m_{2}^{2} \Delta t\left(h_{1}^{2}+h_{2}^{2}+\Delta t^{2}\right)^{2} .
\end{aligned}
$$

If $\Delta t<1$, then

$$
\left\|e^{k+1}\right\|^{2} \leq 2 \alpha_{23} \Delta t \sum_{l=1}^{k}\left\|e^{l}\right\|^{2}+2(k+1)|\Omega| m_{2}^{2} \triangle t\left(h_{1}^{2}+h_{2}^{2}+\triangle t^{2}\right)^{2} .
$$

The Gronwall's inequality yields

$$
\left\|e^{k+1}\right\| \leq \exp \left(\alpha_{23}(k+1) \Delta t\right) \sqrt{\frac{|\Omega|}{\alpha_{23}}} m_{2}\left(h_{1}^{2}+h_{2}^{2}+\triangle t^{2}\right)=C_{21}\left(h_{1}^{2}+h_{2}^{2}+\triangle t^{2}\right) .
$$


For estimating $\left\|\Delta_{h} e^{k+1}\right\|$, we take the inner product of (5.13) with $\delta_{t} e^{k+\frac{1}{2}}=\frac{e^{k+1}-e^{k}}{\Delta t}$, then

$$
\begin{aligned}
& \left\|\frac{e^{k+1}-e^{k}}{\triangle t}\right\|^{2}+\frac{c}{2 \Delta t}\left(\left\|\triangle_{h} e^{k+1}\right\|^{2}-\left\|\triangle_{h} e^{k}\right\|^{2}\right) \\
= & \left\langle\triangle_{h} \mu_{0}\left(\hat{n}^{k+\frac{1}{2}}\right)-\triangle_{h} \mu_{0}\left(\hat{n}^{k+\frac{1}{2}}\right), \delta_{t} e^{k+\frac{1}{2}}\right\rangle+\left\langle\hat{R}^{k+\frac{1}{2}}, \frac{e^{k+1}-e^{k}}{\Delta t}\right\rangle .
\end{aligned}
$$

Similar to the derivation of (5.24), we have

$$
\left\langle\triangle_{h} \mu_{0}\left(\hat{\bar{n}}^{k+\frac{1}{2}}\right)-\triangle_{h} \mu_{0}\left(\hat{n}^{k+\frac{1}{2}}\right), \delta_{t} e^{k+\frac{1}{2}}\right\rangle=\left\langle\mu_{0}\left(\hat{\bar{n}}^{k+\frac{1}{2}}\right)-\mu_{0}\left(\hat{n}^{k+\frac{1}{2}}\right), \delta_{t} \Delta_{h} e^{k+\frac{1}{2}}\right\rangle .
$$

Also, we obtain

$$
\left\langle\hat{R}^{k+\frac{1}{2}}, \frac{e^{k+1}-e^{k}}{\Delta t}\right\rangle \leq \frac{|\Omega| m_{2}^{2}\left(h_{1}^{2}+h_{2}^{2}+\Delta t^{2}\right)^{2}}{2}+\frac{1}{2}\left\|\frac{e^{k+1}-e^{k}}{\Delta t}\right\|^{2} .
$$

Therefore, we have

$$
\begin{aligned}
& \frac{1}{2}\left\|\frac{e^{k+1}-e^{k}}{\Delta t}\right\|^{2}+\frac{c}{2 \Delta t}\left(\left\|\triangle_{h} e^{k+1}\right\|^{2}-\left\|\triangle_{h} e^{k}\right\|^{2}\right) \\
\leq & \left\langle\mu_{0}\left(\hat{\bar{n}}^{k+\frac{1}{2}}\right)-\mu_{0}\left(\hat{n}^{k+\frac{1}{2}}\right), \delta_{t} \Delta_{h} e^{k+\frac{1}{2}}\right\rangle+\frac{|\Omega| m_{2}^{2}\left(h_{1}^{2}+h_{2}^{2}+\Delta t^{2}\right)^{2}}{2} .
\end{aligned}
$$

Replacing the superscript $k$ by $l$ in (5.29) and summing up for $l$ from 0 to $k$, we get

$$
\begin{aligned}
& \frac{1}{2} \sum_{l=0}^{k}\left\|\frac{e^{l+1}-e^{l}}{\Delta t}\right\|^{2}+\frac{c}{2 \Delta t}\left\|\triangle_{h} e^{k+1}\right\|^{2} \\
\leq & \sum_{l=0}^{k}\left\langle\mu_{0}\left(\hat{\bar{n}}^{l+\frac{1}{2}}\right)-\mu_{0}\left(\hat{n}^{l+\frac{1}{2}}\right), \delta_{t} \Delta_{h} e^{l+\frac{1}{2}}\right\rangle+\sum_{l=0}^{k} \frac{|\Omega| m_{2}^{2}\left(h_{1}^{2}+h_{2}^{2}+\Delta t^{2}\right)^{2}}{2} .
\end{aligned}
$$

According to Lemma 3.4, we have

$$
\begin{aligned}
& \sum_{l=0}^{l=k}\left\langle\mu_{0}\left(\hat{\bar{n}}^{l+\frac{1}{2}}\right)-\mu_{0}\left(\hat{n}^{l+\frac{1}{2}}\right), \delta_{t} \Delta_{h} e^{l+\frac{1}{2}}\right\rangle \\
& \quad-\sum_{l=1}^{k}\left\langle\frac{\mu_{0}\left(\hat{\bar{n}}^{l+\frac{1}{2}}\right)-\mu_{0}\left(\hat{n}^{l+\frac{1}{2}}\right)-\left[\mu_{0}\left(\hat{\bar{n}}^{l-\frac{1}{2}}\right)-\mu_{0}\left(\hat{n}^{l-\frac{1}{2}}\right)\right]}{\Delta t}, \Delta_{h} e^{l}\right\rangle \\
& =\frac{1}{\Delta t}\left\langle\mu_{0}\left(\hat{\bar{n}}^{k+\frac{1}{2}}\right)-\mu_{0}\left(\hat{n}^{k+\frac{1}{2}}\right), \Delta_{h} e^{k+1}\right\rangle-\left\langle\frac{\mu_{0}\left(\hat{\bar{n}}^{\frac{5}{2}}\right)-\mu_{0}\left(\hat{n}^{\frac{5}{2}}\right)}{\Delta t}, \Delta_{h} e^{2}\right\rangle \\
& \quad-\sum_{l=3}^{k}\left\langle\frac{\mu_{0}\left(\hat{\bar{n}}^{l+\frac{1}{2}}\right)-\mu_{0}\left(\hat{n}^{l+\frac{1}{2}}\right)-\left(\mu_{0}\left(\hat{\bar{n}}^{l-\frac{1}{2}}\right)-\mu_{0}\left(\hat{n}^{l-\frac{1}{2}}\right)\right)}{\Delta t}, \Delta_{h} e^{l}\right\rangle .
\end{aligned}
$$

Here,

$$
\begin{gathered}
\mu_{0}\left(\hat{\bar{n}}^{k+\frac{1}{2}}\right)-\mu_{0}\left(\hat{n}^{k+\frac{1}{2}}\right)=\mu_{0}^{\prime}\left(\hat{\rho}^{k+\frac{1}{2}} \hat{\bar{n}}^{k+\frac{1}{2}}+\left(1-\hat{\rho}^{k+\frac{1}{2}}\right) \hat{n}^{k+\frac{1}{2}}\right)\left(\hat{\bar{n}}^{k+\frac{1}{2}}-\hat{n}^{k+\frac{1}{2}}\right) \\
=\mu_{0}^{\prime}\left(\hat{\rho}^{k+\frac{1}{2}} \hat{\bar{n}}^{k+\frac{1}{2}}+\left(1-\hat{\rho}^{k+\frac{1}{2}}\right) \hat{n}^{k+\frac{1}{2}}\right)\left(e^{k}+\frac{1}{2} e^{k-1}-\frac{1}{2} e^{k-2}\right)
\end{gathered}
$$




$$
\mu_{0}\left(\hat{\bar{u}}^{\frac{5}{2}}\right)-\mu_{0}\left(\hat{u}^{\frac{5}{2}}\right)=\mu_{0}^{\prime}\left(\hat{\rho}^{\frac{5}{2}} \hat{n}^{\frac{5}{2}}+\left(1-\hat{\rho}^{\frac{5}{2}}\right) \hat{n}^{\frac{5}{2}}\right)\left(e^{2}-e^{1}+\frac{3}{2} e^{1}-\frac{3}{2} e^{0}\right) .
$$

Therefore, we have

$$
\begin{aligned}
& \frac{1}{\Delta t}\left\langle\mu_{0}\left(\hat{\bar{u}}^{k+\frac{1}{2}}\right)-\mu_{0}\left(\hat{u}^{k+\frac{1}{2}}\right), \Delta_{h} e^{k+1}\right\rangle \\
& \quad=\frac{1}{\Delta t}\left\langle\mu_{0}^{\prime}\left(\hat{\rho}^{k+\frac{1}{2}} \hat{\bar{n}}^{k+\frac{1}{2}}+\left(1-\hat{\rho}^{k+\frac{1}{2}}\right) \hat{n}^{k+\frac{1}{2}}\right)\left(e^{k}+\frac{1}{2} e^{k-1}-\frac{1}{2} e^{k-2}\right), \Delta_{h} e^{k+1}\right\rangle \\
& \quad \leq \frac{c}{4 \Delta t}\left\|\Delta_{h} e^{k+1}\right\|^{2}+\frac{2 \alpha_{21}^{2}}{c \Delta t}\left(\left\|e^{k}\right\|^{2}+\frac{1}{2}\left\|e^{k-1}\right\|^{2}+\frac{1}{2}\left\|e^{k-2}\right\|^{2}\right),
\end{aligned}
$$

and

$$
\begin{aligned}
& -\left\langle\frac{\mu_{0}\left(\hat{\bar{u}}^{\frac{5}{2}}\right)-\mu_{0}\left(\hat{u}^{\frac{5}{2}}\right)}{\Delta t}, \Delta_{h} e^{2}\right\rangle \\
= & -\frac{1}{\Delta t}\left\langle\mu_{0}^{\prime}\left(\hat{\rho}^{\frac{5}{2}} \hat{n}^{\frac{5}{2}}+\left(1-\hat{\rho}^{\frac{5}{2}}\right) \hat{n}^{\frac{5}{2}}\right)\left(e^{2}-e^{1}+\frac{3}{2} e^{1}-\frac{3}{2} e^{0}\right), \Delta_{h} e^{2}\right\rangle \\
\leq & \alpha_{21}\left\|\frac{e^{2}-e^{1}}{\Delta t}+\frac{3}{2} \frac{e^{1}-e^{0}}{\Delta t}\right\|\left\|\Delta_{h} e^{2}\right\| \leq \frac{1}{4}\left[\left\|\frac{e^{2}-e^{1}}{\Delta t}\right\|^{2}+\left\|\frac{e^{1}-e^{0}}{\Delta t}\right\|^{2}\right]+\frac{13 \alpha_{21}^{2}}{4}\left\|\Delta_{h} e^{2}\right\|^{2} .
\end{aligned}
$$

Its follows from Lemma 3.5 that

$$
\begin{aligned}
& \frac{1}{\Delta t}\left[\mu_{0}\left(\hat{\bar{n}}^{l+\frac{1}{2}}\right)-\mu_{0}\left(\hat{n}^{l+\frac{1}{2}}\right)-\left(\mu_{0}\left(\hat{\bar{n}}^{l-\frac{1}{2}}\right)-\mu_{0}\left(\hat{n}^{l-\frac{1}{2}}\right)\right)\right] \\
=\mu_{0}^{\prime}\left(\hat{\rho}^{l} \hat{n}^{l+\frac{1}{2}}+\left(1-\hat{\rho}^{l}\right) \hat{n}^{l-\frac{1}{2}}\right) \frac{e^{l}-e^{l-1}+\frac{1}{2}\left(e^{l-1}-e^{l-2}\right)-\frac{1}{2}\left(e^{l-2}-e^{l-3}\right)}{\Delta t} & \\
& \quad+\mu_{0}^{\prime \prime}\left(\hat{\xi}^{l}\right)\left(\hat{\rho}^{l} \hat{e}^{l+\frac{1}{2}}+\left(1-\hat{\rho}^{l}\right) \hat{e}^{l-\frac{1}{2}}\right) \frac{\bar{n}^{l}-\bar{n}^{l-1}+\frac{1}{2}\left(\bar{n}^{l-1}-\bar{n}^{l-2}\right)-\frac{1}{2}\left(\bar{n}^{l-2}-\bar{n}^{l-3}\right)}{\Delta t},
\end{aligned}
$$

where $\hat{\rho}^{l} \in(0,1)$ and $\hat{\xi}^{l} \in\left(\min \left\{\gamma_{1}, \gamma_{2}\right\}, \max \left\{\gamma_{1}, \gamma_{2}\right\}\right)$. Consequently, we have

$$
\begin{aligned}
& -\sum_{l=3}^{k}\left\langle\frac{\mu_{0}\left(\hat{\bar{n}}^{l+\frac{1}{2}}\right)-\mu_{0}\left(\hat{n}^{l+\frac{1}{2}}\right)-\left(\mu_{0}\left(\hat{\bar{n}}^{l-\frac{1}{2}}\right)-\mu_{0}\left(\hat{n}^{l-\frac{1}{2}}\right)\right)}{\Delta t}, \Delta_{h} e^{l}\right\rangle \\
=- & \sum_{l=3}^{k}\left\langle\mu_{0}^{\prime}\left(\hat{\rho}^{l} \hat{n}^{l+\frac{1}{2}}+\left(1-\hat{\rho}^{l}\right) \hat{n}^{l-\frac{1}{2}}\right) \frac{e^{l}-e^{l-1}+\frac{1}{2}\left(e^{l-1}-e^{l-2}\right)-\frac{1}{2}\left(e^{l-2}-e^{l-3}\right)}{\Delta t}, \Delta_{h} e^{l}\right\rangle \\
& -\sum_{l=3}^{k}\left\langle\mu_{0}^{\prime \prime}\left(\xi^{l}\right)\left(\hat{\rho}^{l} \hat{e}^{l+\frac{1}{2}}+\left(1-\hat{\rho}^{l}\right) \hat{e}^{l-\frac{1}{2}}\right) \frac{\bar{n}^{l}-\bar{n}^{l-1}+\frac{\bar{n}^{l-1}-\bar{n}^{l-2}}{2}-\frac{\bar{n}^{l-2}-\bar{n}^{l-3}}{2}}{\Delta t}, \Delta_{h} e^{l}\right\rangle .
\end{aligned}
$$

The first term of the right hand side of (5.31) satisfies

$$
\begin{gathered}
-\sum_{l=3}^{k}\left\langle\mu_{0}^{\prime}\left(\hat{\rho}^{l} \hat{n}^{l+\frac{1}{2}}+\left(1-\hat{\rho}^{l}\right) \hat{n}^{l-\frac{1}{2}}\right) \frac{e^{l}-e^{l-1}+\frac{1}{2}\left(e^{l-1}-e^{l-2}\right)-\frac{1}{2}\left(e^{l-2}-e^{l-3}\right)}{\Delta t}, \Delta_{h} e^{l}\right\rangle \\
\leq \frac{1}{4} \sum_{l=3}^{k}\left\|\frac{e^{l}-e^{l-1}}{\Delta t}\right\|^{2}+\alpha_{21}^{2} \sum_{l=3}^{k}\left\|\Delta_{h} e^{l}\right\|^{2}+\frac{1}{8} \sum_{l=3}^{k}\left\|\frac{e^{l-1}-e^{l-2}}{\Delta t}\right\|^{2} \\
+\frac{\alpha_{21}^{2}}{2} \sum_{l=3}^{k}\left\|\Delta_{h} e^{l}\right\|^{2}+\frac{1}{8} \sum_{l=3}^{k}\left\|\frac{e^{l-2}-e^{l-3}}{\Delta t}\right\|^{2}+\frac{\alpha_{21}^{2}}{2} \sum_{l=3}^{k}\left\|\Delta_{h} e^{l}\right\|^{2} .
\end{gathered}
$$


As for the second term of (5.31), we have

$$
\begin{aligned}
& \left|\sum_{l=3}^{k}\left\langle\mu_{0}^{\prime \prime}\left(\xi^{l}\right)\left(\hat{\rho}^{l} \hat{e}^{l+\frac{1}{2}}+\left(1-\hat{\rho}^{l}\right) \hat{e}^{l-\frac{1}{2}}\right) \frac{\bar{n}^{l}-\bar{n}^{l-1}+\frac{\bar{n}^{l-1}-\bar{n}^{l-2}}{2}-\frac{\bar{n}^{l-2}-\bar{n}^{l-3}}{2}}{\Delta t}, \Delta_{h} e^{l}\right\rangle\right| \\
\leq & 2 \sum_{l=3}^{k} \alpha_{22} \alpha_{24}\left[\left\|\hat{\rho} e^{l}\right\|+\left\|\left(1-\frac{\hat{\rho}}{2}\right) e^{l-1}\right\|+\left\|\left(\frac{1}{2}-\hat{\rho}\right) e^{l-2}\right\|+\left\|\frac{1-\hat{\rho}}{2} e^{l-3}\right\|\right]\left\|\Delta_{h} e^{l}\right\| \\
= & \sum_{l=3}^{k} \alpha_{22} \alpha_{24}\left\|e^{l}\right\|^{2}+\sum_{l=2}^{k-1} \alpha_{22} \alpha_{24}\left\|e^{l}\right\|^{2}+\sum_{l=1}^{k-2} \frac{\alpha_{22} \alpha_{24}}{4}\left\|e^{l}\right\|^{2} \\
& +\sum_{l=1}^{k-3} \frac{\alpha_{22} \alpha_{24}}{4}\left\|e^{l}\right\|^{2}+4 \alpha_{22} \alpha_{24} \sum_{l=3}^{k}\left\|\Delta_{h} e^{l}\right\|^{2} .
\end{aligned}
$$

Hence,

$$
\begin{aligned}
& \sum_{l=0}^{l=k}\left\langle\mu_{0}\left(\hat{\bar{u}}^{l+\frac{1}{2}}\right)-\mu_{0}\left(\hat{u}^{l+\frac{1}{2}}\right), \delta_{t} \Delta_{h} e^{l+\frac{1}{2}}\right\rangle \\
= & \frac{1}{\Delta t}\left\langle\mu_{0}\left(\hat{\bar{u}}^{k+\frac{1}{2}}\right)-\mu_{0}\left(\hat{u}^{k+\frac{1}{2}}\right), \Delta_{h} e^{k+1}\right\rangle-\left\langle\frac{\mu_{0}\left(\hat{\bar{u}}^{\frac{5}{2}}\right)-\mu_{0}\left(\hat{u}^{\frac{5}{2}}\right)}{\Delta t}, \Delta_{h} e^{2}\right\rangle \\
& -\sum_{l=3}^{k}\left\langle\frac{\mu_{0}\left(\hat{\bar{u}}^{l+\frac{1}{2}}\right)-\mu_{0}\left(\hat{u}^{l+\frac{1}{2}}\right)-\left(\mu_{0}\left(\hat{\bar{u}}^{l-\frac{1}{2}}\right)-\mu_{0}\left(\hat{u}^{l-\frac{1}{2}}\right)\right)}{\Delta t}, \Delta_{h} e^{l}\right\rangle \\
\leq & \frac{c}{4 \Delta t}\left\|\Delta_{h} e^{k+1}\right\|^{2}+\frac{2 \alpha_{21}^{2}}{c \Delta t}\left(\left\|e^{k}\right\|^{2}+\frac{1}{4}\left\|e^{k-1}\right\|^{2}+\frac{1}{4}\left\|e^{k-2}\right\|^{2}\right) \\
& +\frac{1}{2} \sum_{l=0}^{k-1}\left\|\frac{e^{l+1}-e^{l}\left\|^{2}+\alpha_{26} \sum_{l=2}^{k}\right\| \Delta_{h} e^{l} \|^{2}}{\Delta t}\right\|\left\|_{l=3}^{k} \alpha_{22} \alpha_{24}\right\| e^{l}\left\|^{2}+\sum_{l=2}^{k-1} \alpha_{22} \alpha_{24}\right\| e^{l}\left\|^{2}+\sum_{l=1}^{k-2} \frac{\alpha_{22} \alpha_{24}}{4}\right\| e^{l}\left\|^{2}+\sum_{l=1}^{k-3} \frac{\alpha_{22} \alpha_{24}}{4}\right\| e^{l} \|^{2} .
\end{aligned}
$$

Combining (5.33) with (5.30), we get

$$
\begin{aligned}
& \frac{1}{2} \sum_{l=0}^{k}\left\|\frac{e^{l+1}-e^{l}}{\Delta t}\right\|^{2}+\frac{c}{2 \Delta t}\left\|\Delta_{h} e^{k+1}\right\|^{2} \\
\leq & \frac{c}{4 \Delta t}\left\|\Delta_{h} e^{k+1}\right\|^{2}+\frac{2 \alpha_{21}^{2}}{c \Delta t}\left(\left\|e^{k}\right\|^{2}+\frac{1}{2}\left\|e^{k-1}\right\|^{2}+\frac{1}{2}\left\|e^{k-2}\right\|^{2}\right) \\
& +\frac{1}{2} \sum_{l=0}^{k-1}\left\|\frac{e^{l+1}-e^{l}}{\Delta t}\right\|^{2}+\alpha_{26} \sum_{l=2}^{k}\left\|\Delta_{h} e^{l}\right\|^{2}+\sum_{l=3}^{k} \alpha_{22} \alpha_{24}\left\|e^{l}\right\|^{2}+\sum_{l=2}^{k-1} \alpha_{22} \alpha_{24}\left\|^{l}\right\|^{2} \\
& +\sum_{l=1}^{k-2} \frac{\alpha_{22} \alpha_{24}}{4}\left\|e^{l}\right\|^{2}+\sum_{l=1}^{k-3} \frac{\alpha_{22} \alpha_{24}}{4}\left\|e^{l}\right\|^{2}+\sum_{l=0}^{k} \frac{|\Omega| m_{2}^{2}\left(h_{1}^{2}+h_{2}^{2}+\Delta t^{2}\right)^{2}}{2} .
\end{aligned}
$$

Therefore,

$$
\begin{array}{r}
\frac{c}{4 \Delta t}\left\|\triangle_{h} e^{k+1}\right\|^{2} \leq \frac{2 \alpha_{21}^{2}}{c \Delta t}\left(\left\|e^{k}\right\|^{2}+\frac{1}{4}\left\|e^{k-1}\right\|^{2}+\frac{1}{4}\left\|e^{k-2}\right\|^{2}\right)+\alpha_{26} \sum_{l=2}^{k}\left\|\Delta_{h} e^{l}\right\|^{2} \\
+\sum_{l=3}^{k} \alpha_{22} \alpha_{24}\left\|e^{l}\right\|^{2}+\sum_{l=2}^{k-1} \alpha_{22} \alpha_{24}\left\|e^{l}\right\|^{2}+\sum_{l=1}^{k-2} \frac{\alpha_{22} \alpha_{24}}{4}\left\|e^{l}\right\|^{2}
\end{array}
$$




$$
+\sum_{l=1}^{k-3} \frac{\alpha_{22} \alpha_{24}}{4}\left\|e^{l}\right\|^{2}+\sum_{l=0}^{k} \frac{|\Omega| m_{2}^{2}\left(h_{1}^{2}+h_{2}^{2}+\Delta t^{2}\right)^{2}}{2} .
$$

Accordingly,

$$
\begin{aligned}
\left\|\triangle_{h} e^{k+1}\right\|^{2} \leq \frac{8 \alpha_{21}^{2}}{c^{2}} & \left(\left\|e^{k}\right\|^{2}+\frac{1}{2}\left\|e^{k-1}\right\|^{2}+\frac{1}{2}\left\|e^{k-2}\right\|^{2}\right)+\frac{4 \alpha_{26}}{c} \Delta t \sum_{l=2}^{k}\left\|\Delta_{h} e^{l}\right\|^{2} \\
& +\sum_{l=3}^{k} \frac{4 \alpha_{22} \alpha_{24}}{c} \Delta t\left\|e^{l}\right\|^{2}+\sum_{l=2}^{k-1} \frac{4 \alpha_{22} \alpha_{24}}{c} \Delta t\left\|e^{l}\right\|^{2}+\sum_{l=1}^{k-2} \frac{\alpha_{22} \alpha_{24}}{c} \Delta t\left\|e^{l}\right\|^{2} \\
& +\sum_{l=1}^{k-3} \frac{\alpha_{22} \alpha_{24}}{c} \Delta t\left\|e^{l}\right\|^{2}+\sum_{l=0}^{k} \frac{2|\Omega| m_{2}^{2}\left(h_{1}^{2}+h_{2}^{2}+\Delta t^{2}\right)^{2}}{c} \Delta t
\end{aligned}
$$

According to (5.27), if $\Delta t<1$, we get

$$
\left\|e^{k+1}\right\|^{2} \leq 2 \alpha_{23} \Delta t \sum_{l=1}^{k}\left\|e^{l}\right\|^{2}+2(k+1)|\Omega| m_{2}^{2} \triangle t\left(h_{1}^{2}+h_{2}^{2}+\triangle t^{2}\right)^{2} .
$$

Combining the above results, (5.27) with (5.36), we have

$$
\begin{aligned}
\left\|e^{k+1}\right\|^{2}+\left\|\triangle_{h} e^{k+1}\right\|^{2} & \\
\leq 2 \alpha_{23} \Delta & t \sum_{l=1}^{k}\left\|e^{l}\right\|^{2}+2(k+1)|\Omega| m_{2}^{2} \Delta t\left(h_{1}^{2}+h_{2}^{2}+\Delta t^{2}\right)^{2}+\frac{4 \alpha_{26}}{c} \Delta t \sum_{l=2}^{k}\left\|\Delta_{h} e^{l}\right\|^{2} \\
& +\frac{8 \alpha_{21}^{2}}{c^{2}}\left[2 \alpha_{23} \Delta t \sum_{l=1}^{k-1}\left\|e^{l}\right\|^{2}+2 k|\Omega| m_{2}^{2} \Delta t\left(h_{1}^{2}+h_{2}^{2}+\Delta t^{2}\right)^{2}\right] \\
& +\frac{4 \alpha_{21}^{2}}{c^{2}}\left[2 \alpha_{23} \Delta t \sum_{l=1}^{k-2}\left\|e^{l}\right\|^{2}+2(k-1)|\Omega| m_{2}^{2} \Delta t\left(h_{1}^{2}+h_{2}^{2}+\Delta t^{2}\right)^{2}\right] \\
& +\frac{4 \alpha_{21}^{2}}{c^{2}}\left[2 \alpha_{23} \Delta t \sum_{l=1}^{k-3}\left\|e^{l}\right\|^{2}+2(k-2)|\Omega| m_{2}^{2} \Delta t\left(h_{1}^{2}+h_{2}^{2}+\Delta t^{2}\right)^{2}\right] \\
& +\sum_{l=3}^{k} \frac{4 \alpha_{22} \alpha_{24}}{c} \Delta t\left\|e^{l}\right\|^{2}+\sum_{l=2}^{k-1} \frac{4 \alpha_{22} \alpha_{24}}{c} \Delta t\left\|e^{l}\right\|^{2}+\sum_{l=1}^{k-2} \frac{\alpha_{22} \alpha_{24}}{c} \Delta t\left\|e^{l}\right\|^{2} \\
& +\sum_{l=1}^{k-3} \frac{\alpha_{22} \alpha_{24}}{c} \Delta t\left\|e^{l}\right\|^{2}+\sum_{l=0}^{k} \frac{2|\Omega| m_{2}^{2}\left(h_{1}^{2}+h_{2}^{2}+\Delta t^{2}\right)^{2}}{c} \Delta t \\
\leq\left(2 \alpha_{23}\right. & \left.+\frac{32 \alpha_{23} \alpha_{21}^{2}}{c^{2}}+\frac{10 \alpha_{22} \alpha_{24}}{c}\right) \Delta t \sum_{l=1}^{k}\left\|e^{l}\right\|^{2}+\frac{4 \alpha_{26}}{c} \Delta t \sum_{l=1}^{k}\left\|\Delta_{h} e^{l}\right\|^{2} \\
& +(k+1)\left(2+\frac{32 \alpha_{21}^{2}}{c^{2}}+\frac{2}{c}\right)|\Omega| \alpha_{22}^{2}\left(h_{1}^{2}+h_{2}^{2}+\Delta t^{2}\right)^{2} \Delta t \\
\leq 2 \alpha_{27} \Delta & t \sum_{l=1}^{k}\left(\left\|e^{l}\right\|^{2}+\left\|\Delta e^{l}\right\|^{2}\right)+2(k+1) \alpha_{25}|\Omega| m_{2}^{2}\left(h_{1}^{2}+h_{2}^{2}+\Delta t^{2}\right)^{2} \Delta t .
\end{aligned}
$$

Using the Gronwall's inequality yields

$$
\left\|e^{k+1}\right\|^{2}+\left\|\triangle_{h} e^{k+1}\right\|^{2} \leq \frac{\alpha_{25}}{\alpha_{27}} \exp \left(\alpha_{27}(k+1) \Delta t\right) \cdot|\Omega|\left[m_{2}\left(h_{1}^{2}+h_{2}^{2}+\Delta t^{2}\right)\right]^{2},
$$


where $k=0,1, \ldots, K-1$. Using Lemma 3.3 and (5.28), we can get

$$
\begin{aligned}
\left\|e^{k+1}\right\|_{\infty}^{2} & \leq k_{0}\left\|e^{k+1}\right\|\left(\left\|\triangle_{h} e^{k+1}\right\|+\left\|e^{k+1}\right\|\right) \leq k_{0} \sqrt{2\left\|e^{k+1}\right\|^{2}\left(\left\|e^{k+1}\right\|^{2}+\left\|\triangle_{h} e^{k+1}\right\|^{2}\right)} \\
& \leq k_{0} \sqrt{\frac{2 \alpha_{25}}{\alpha_{23} \alpha_{27}}} \exp \left(\left(\alpha_{23}+\alpha_{27}\right)(k+1) \Delta t\right) m_{2}^{2}|\Omega|\left(h_{1}^{2}+h_{2}^{2}+\Delta t^{2}\right)^{2} .
\end{aligned}
$$

Therefore,

$$
\left\|e^{k+1}\right\|_{\infty} \leq C_{22}\left(h_{1}^{2}+h_{2}^{2}+\triangle t^{2}\right)
$$

\section{A Numerical Example}

In this section, we use the two second order numerical schemes to obtain the steady state of the fourth order parabolic equations (2.5a)-(2.5b). The substance isobutane $(\mathrm{nC} 4)$ is selected for comparing with the results of second order parabolic equation provided in [22]. The critical properties and the normal boiling point of this substance are provided in Table 6.1. The parameters $a(T), b$ and $c(T)$ are calculated by the formulas (2.2) and (2.3). For comparison with the results in [22], all the following numerical results are implementated on the domain $\Omega=[0, L]^{2}$ with $L=2 \times 10^{-8}$ meters. The initial condition is set as: the molar density equals the liquid isobutane under a saturated pressure in the region $[0.3 L, 0.7 L]$, the rest of the domain is filled with a saturated isobutane gas. The periodic boundary condition is imposed.

Table 6.1: Critical properties (Data from Table 3.1 of the book of Firoozabadi [6]), $\omega$ and $m$ (our computed results) of the selected substance, isobutane (nC4).

\begin{tabular}{|c|c|c|c|c|}
\hline symbol & $T_{c}, K$ & $P_{c}$ & $\omega$ & $\mathrm{m}$ \\
\hline$n \mathrm{C}_{4}$ & 425.18 & $3.797 \mathrm{MPa}$ & 0.1990 & 0.6709 \\
\hline
\end{tabular}

\subsection{Numerical accuracy test}

In this subsection, we check the convergence rate in time of the Crank-Nicolson scheme (4.4)-(4.5) and the second order linearized scheme (5.4a)-(5.4b). Here, the exact solution is defined by the molar density at $T_{m}=10^{-7}$ obtained from $\Delta t=10^{-11}$ on a $400 \times 400$ meshes on the domain $\Omega$. The $l^{2}$ norm of errors at several time points and the convergence rate of these schemes are provided by Table 6.2 .

Table 6.2: The temporal convergence of the two schemes at $T_{m}=10^{-7}$. (a) The Crank-Nicolson Scheme; (b) The linearized scheme.

\begin{tabular}{|c|c|c|}
\hline$\Delta t$ & $l^{2}$ error & rate of convergence \\
\hline $1.0 \mathrm{e}-10$ & $2.7107 \mathrm{e}-5$ & - \\
\hline $2.0 \mathrm{e}-10$ & $1.0925 \mathrm{e}-4$ & 2.0109 \\
\hline $4.0 \mathrm{e}-10$ & $4.3783 \mathrm{e}-4$ & 2.0027 \\
\hline $8.0 \mathrm{e}-10$ & 0.0018 & 2.0396 \\
\hline $1.0 \mathrm{e}-9$ & 0.0027 & 2.0002 \\
\hline
\end{tabular}

(a)

\begin{tabular}{|c|c|c|}
\hline$\Delta t$ & $l^{2}$ error & rate of convergence \\
\hline $1.0 \mathrm{e}-10$ & 0.0022 & - \\
\hline $2.0 \mathrm{e}-10$ & 0.0047 & 1.0952 \\
\hline $4.0 \mathrm{e}-10$ & 0.0096 & 1.0304 \\
\hline $8.0 \mathrm{e}-10$ & 0.0194 & 1.0150 \\
\hline $1.0 \mathrm{e}-9$ & 0.0244 & 1.0253 \\
\hline
\end{tabular}

(b)

It is clear that the Crank-Nicolson scheme has exactly second order temporal convergence rate and the linearized scheme has only first order temporal convergence rate. The loss of the 


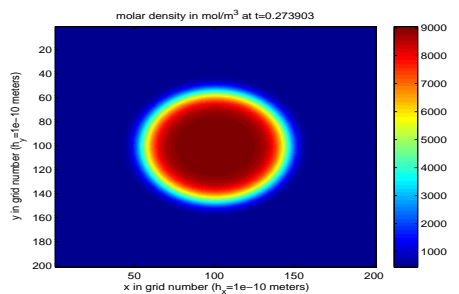

(a1)

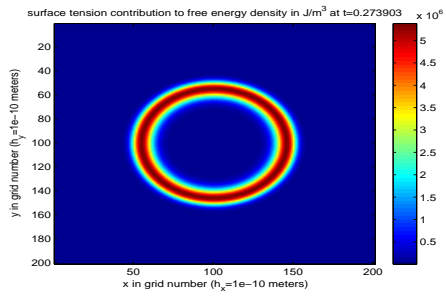

(b1)

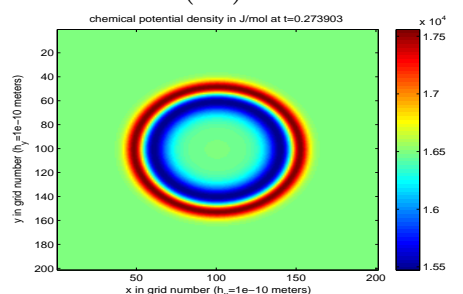

(c1)

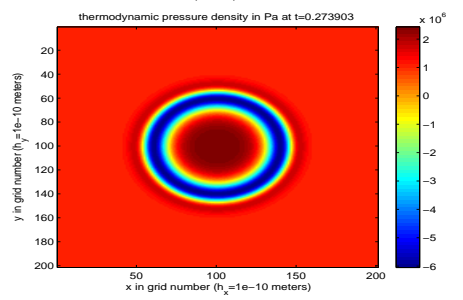

(d1)

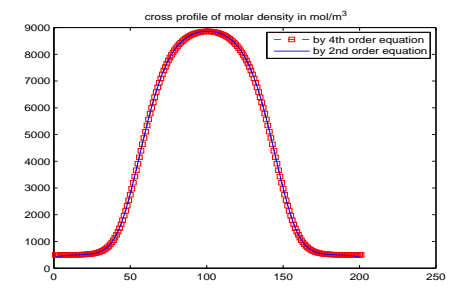

(a2)

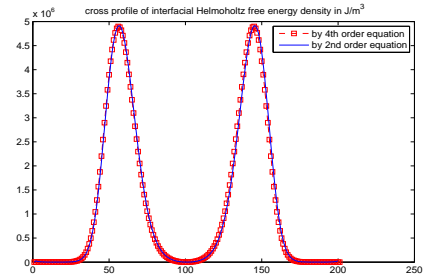

(b2)

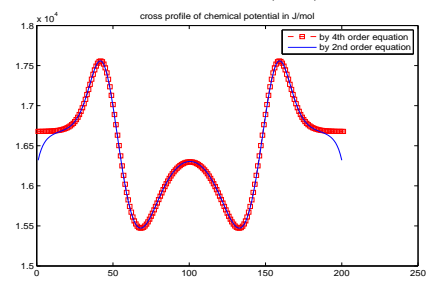

(c2)

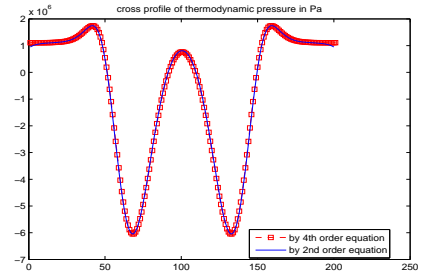

(d2)

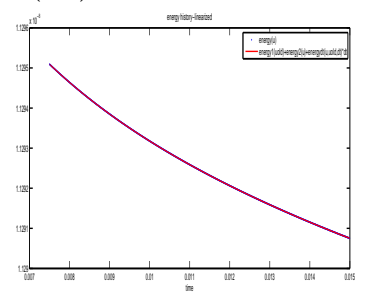

(e3)

(e1)

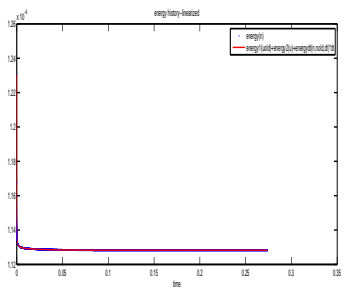

(e2)

Fig. 6.1. Numerical results given by the fourth order equation at steady state: (a1) molar density, (b1) interfacial Helmholtz free energy density, (c1) chemical potential density, (d1) thermodynamic pressure density, cross profile of molar (a2), interfacial Helmholtz free energy (b2), chemical potential (c2), thermodynamic pressure (d2) density; total energy evolution history given by second order equation (e1), fourth order equation (e2), current energy evolution history given by fourth-order equation.

convergence rate of the linearized scheme may arise from its large truncation error. However, at every time step, the Crank-Nicolson scheme takes several steps of the Newton iteration due 
to its nonlinear character, while the linearized scheme does not have this extra cost. So it is worthy of carrying out the long time simulation with the linearized scheme.

\subsection{Spatial distribution of molar density and other chemical properties}

Here, the homogeneous contribution of chemical potential $\mu_{0}$ is defined as (2.6b). The surface tension contribution to the Helmholtz free energy density $f_{\text {intfTens }}$ is also defied as [22]

$$
f_{\text {intfTens }}=2 f_{\nabla}(n)=c \nabla n \cdot \nabla n .
$$

And the thermodynamic pressure $p_{0}$ is defined as [22]

$$
\begin{aligned}
& p_{0}=n\left(\frac{\partial f_{0}}{\partial n}\right)-f_{0}=n \mu_{0}-f_{0} \\
= & \frac{n R T}{1-b n}-\frac{n^{2} a(T)}{1+2 b n-b^{2} n^{2}}=\frac{R T}{v-b}-\frac{a(T)}{v(v+b)+b(v-b)} .
\end{aligned}
$$

Since the evolution history and the steady state obtained from the two schemes are very close, the results offered by either one can illustrate the same phenomenon. Hereafter, we use the solution obtained from the linearized scheme to calculate all the variables of interest. The whole domain $\Omega$ is discretized by $200 \times 200$ rectangular meshes. Figure 6.1 provides the molar density, interfacial Helmholtz free energy density, homogeneous chemical potential, the thermodynamic pressure and the total energy evolution history calculated from the solution of the four-order equation. The steady state is defined by the condition that the relative error between two neighboring time step is less than $10^{-8}$. From Figure 6.1, we can see that the fourth-order parabolic equation can describe dramatically changing of $n, f_{\text {intTens }}, \mu_{0}, p_{0}$ and guarantee the energy decreasing property.

\subsection{Calculation of interface tension and verification against Young-Laplace eqn}

The surface tension $\sigma$ is defined as the net contractive force per unit length with a unit of $N / m$ mechanically or the work for creating a unit area of interface with a unit of $J / m^{2}$. Here

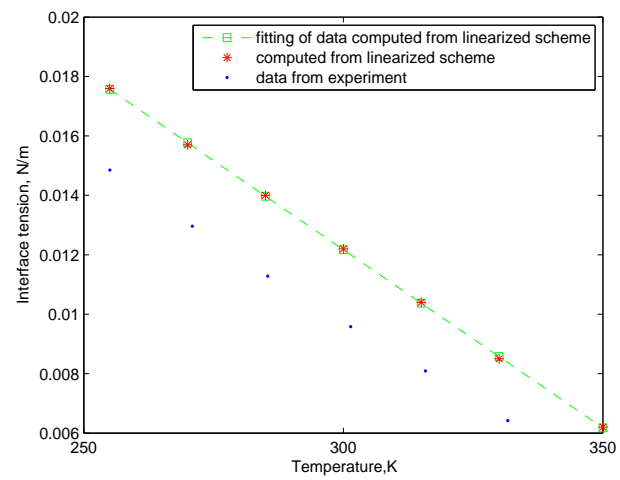

(a)

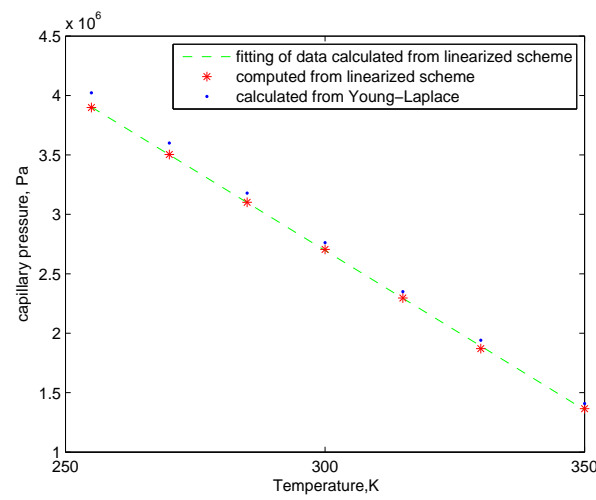

(b)

Fig. 6.2. Comparison between numerical predictions given by second order linearized scheme and laboratory data: (a) surface tension $(\mathrm{N} / \mathrm{m})$; (b) capillary pressure. 
we use the formula $[6,22]$

$$
\sigma=\frac{\partial F}{\partial A}=\frac{F(n)-F_{0}\left(n_{\text {bulk }}\right)}{A} .
$$

with the assumption that $\sigma$ is spatially constant within the interface for the given system. The numerator term of the equation (6.3) evaluates the contribution of the surface tension to the total free energy at equilibrium state. Here, the volume of the liquid droplet is assumed as a constant all the time and becomes a perfect circle at steady state. The radius of the droplet is also $r=2 \times 10^{-8} \times(0.16 / \pi)^{1 / 2}=4.514 \times 10^{-9}$. The length of the circle $A=2 \pi \times 4,514 \times 10^{-9}$ meters. The surface tension ranging from $250 \mathrm{~K}$ to $350 \mathrm{~K}$ are plotted in Figure 6.2 (a) comparing with the laboratory results provided in Table 2 of [16]. We can see that, the difference between the surface tension trend calculated by the steady state of our fourth order equation and the experimental data is small from the engineering point of view.

The pressure is calculated by the well-known Young-Laplace equation as $P_{c}=P_{\text {liquid }}-$ $P_{\text {gas }}=\sigma / r[6,22]$. The pressure of liquid drop $P_{\text {liquid }}$ is picked from the central grid point $(101,101)$ and the pressure of gas region $P_{\text {gas }}$ is calculated at the point $(51,51)$. The difference between the two is the capillary pressure $p_{0}=P_{\text {liquid }}-P_{\text {gas }}$. On the other hand, the capillary pressure predicted by Young-Laplace equation is $p=\sigma / r$. The capillary pressure $P_{c}$ from temperature $250 \mathrm{~K}$ to $350 \mathrm{~K}$ obtained from these two methods are plotted in Figure 6.2 (b). These two methods agree with errors around or smaller than $6 \%$.

\section{Conclusions}

In this paper, we have derived the fourth-order parabolic equation on a two-dimensional rectangular domain $\Omega$ and provided two second order energy stable schemes to solve it. Here, we should point out that, our energy stability was obtained on an modified form of the original total discrete energy. There is still a minor gap between our modified energy stability and the direct discretization in terms of the original variable $n$. To make up this gap is left as one aspect of our future work. The mass conservation, energy decreasing property, and the convergence of these two schemes are provided. To overcome the difficulty in deriving the convergence of these two schemes, which contributes to the possible unboundness of the chemical potential $\mu_{0}$ and its derivative, $\mu_{0}^{\prime}$, on the definitional domain $(0,1 / b)$, we impose a reasonable hypothesis that the exact solution to the fourth-order equation and the numerical solution to these two schemes at every step are in a subset of $(0,1 / b)$, which is in the form of $[\theta, 1 / b-\theta]$. The numerical results showed in the above section demonstrate the effectiveness of the fourth-order equation and these two schemes.

Acknowledgments. We are grateful to Prof. Zhizhong Sun of Department of Mathematics of Southeast University and Prof. Hehu Xie of Institute of Computational Mathematics of Chinese Academy of Sciences for providing useful suggestions and many helpful discussions. The research of Zhonghua Qiao is partially supported by the Hong Kong Research Grant Council GRF grant 15302214, NSFC/RGC Joint Research Scheme N_HKBU204/12 and the Hong Kong Polytechnic University internal grant 1-ZE33. Shuyu Sun gratefully acknowledges that the research reported in this publication was supported by funding from King Abdullah University of Science and Technology (KAUST). 


\section{References}

[1] G.D. Akrivis, Finite difference discretization of the cubic Schrödinger equation. IMA J. Numer. Anal., 13 (1993), 115-124.

[2] G.D. Akrivis, V.A. Dogalis and O.A. Karakashina, On fully discrete Galerkin methods of secondorder temporal accuracy for the nonlinear Schrödinger equarion. Numer. Math., 59 (1991), 31-53.

[3] K. Bao, Y. Shi, S. Sun and X.P. Wang, A finite element method for the numerical solution of the coupled Cahn-Hilliard and Navier-Stokes system for moving contact line problems, J. Comput. Phys., 231 (2012), 8083-8099.

[4] B. Breure and C.J. Peters, Modeling of the surface tension of pure components and mixtures using the density gradient theory combined with a theoretically derived influence parameter correlation, Fluid Phase Equilib., 34 (2012), 189-196.

[5] C. Dawson, S. Sun and M.F. Wheeler, Compatible algorithms for coupled flow and transport, Comput. Meth. Appl. Mech. Eng., 193 (2004), 2565-2580.

[6] A. Firoozabadi, Thermodynamics of hydrocarbon reservoirs, McGraw-Hill, New York, 1999.

[7] F. Guillén-González and G. Tierra, On linear schemes for a Cahn-Hilliard diffuse interface model, J. Comput. Phys., 234 (2013), 140-171.

[8] R.H. Guo, L.Y. Ji and Y. Xu, High order local discontinuous Galerkin methods for the Allen-Cahn equation: Analysis and simulation, J. Comput. Math., 34 (2016), 135-158.

[9] K.B. Haugen and A. Firoozabadi, Composition at the interface between multicomponent nonequilibrium fluid phases, J. Chem. Phys., 130 (2009), 064707.

[10] J. Kou and S. Sun, An adaptive finite element method for simulating surface tension with the gradient theory of fluid interfaces, J. Comput. Appl. Math., 255 (2014), 593-604.

[11] C. Lee, D. Jeong, J. Shin, Y. Li and J. Kim, A fourth-order spatial accurate and practically stable compact scheme for the Cahn-Hilliard equation, Physica A., 409 (2014), 17-28.

[12] D. Li and Z.H. Qiao, On second order semi-implicit Fourier spectral methods for 2D Cahn-Hilliard equations, J. Sci. Comput., 70 (2017), 301-341.

[13] D. Li and Z.H. Qiao, On the stabilization size of semi-implicit Fourier-spectral methods for 3D Cahn-Hilliard equations, Commun. Math. Sci., 15 (2017), 1489-1506.

[14] D. Li, Z.H. Qiao and T. Tang, Characterizing the stabilization size for semi-implicit Fourierspectral method to phase field equations, SIAM J. Numer. Anal., 54 (2016), 1653-1681.

[15] J. Li, Z.Z. Sun and X. Zhao, A three level linearized compact difference scheme for the CahnHilliard equation, Sci. China Math., 55 (2012), 805-826.

[16] H. Lin and Y.Y. Duan, Surface tension measurements of propane (R-290) and isobutane (R- 600a) from (253 to 333) K, J. Chem. Eng. Data, 48 (2003), 1360-1363.

[17] F.S. Luo, T. Tang and H.H. Xie, Parameter-free time adaptivity based on energy evolution for the Cahn-Hilliard equation, Commun. Comput. Phys., 19 (2016), 1542-1563.

[18] C. Miqueu, B. Mendiboure, A. Graciaa and J. Lachaise, Modelling of the surface tension of pure components with the gradient theory of fluid interfaces: a simple and accurate expression for the influence parameters, Fluid Phase Equilib., 207 (2003), 225-246.

[19] C. Miqueu, B. Mendiboure, A. Graciaa, and J. Lachaise, Modeling of the surface tension of multicomponent mixtures with the gradient theory of fluid interfaces, Ind. Eng. Chem. Res., 44 (2005), 3321-3329.

[20] A. Onuki, Phase Transition Dynamics, Cambridge University Press, 2002.

[21] D. Y. Peng and D. B. Robinson, A new two-constant equation of state, Ind. Eng. Chem. Fundam., 15 (1976), 59-64.

[22] Z. Qiao and S. Sun, Two-phase fluid simulation using a diffuse interface model with Peng-Robinson equation of state, SIAM J. Sci. Comput., 36 (2014), B708-B728.

[23] Z. Qiao, Z. Sun and Z. Zhang, The stability and convergence of two linearized finite difference schemes for the nonlinear epitaxial growth model, Numer. Meth. PDE, 28 (2012), 1893-1915. 
[24] Z. Qiao, Z. Sun and Z. Zhang, Stability and convergence of second-order schemes for the nonlinear epitaxial growth model without slope selection, Math. Comp., 84 (2015), 653-674.

[25] Z. Qiao, Z. Zhang and T. Tang, An adaptive time-stepping strategy for the molecular beam epitaxy models, SIAM J. Sci. Comput., 33 (2011), 1395-1414.

[26] Z.H. Qiao, C. Wang, S.M. Wise and Z.R. Zhang, Error analysis of a finite difference scheme for the epitaxial thin film model with slope selection with an improved convergence constant, Inter. J. Numer. Anal. Mod., 14 (2017), 283-305.

[27] L. Rongy, K.B. Haugen and A. Firoozabadi, Mixing from fickian diffusion and natural convection in binary non-equilibrium fluid phases, AIChE J., 58 (2012), 1336-1345.

[28] J. Shen, C. Wang, X. Wang and S.M. Wise, Second-order convex splitting schemes for gradient flows with ehrlich-schwoebel type energy: Application to thin film epitaxy, SIAM J. Numer. Anal., 50 (2012), 105-125.

[29] J. Shen and X. Yang, Numerical approximations of Allen-cahn and Cahn-Hilliard equations, Disc. Cont. Dyn.. Syst. A, 28 (2010), 1669-1691.

[30] S. Sun and J. Geiser, Multiscale discontinuous galerkin and operator-splitting methods for modeling subsurface flow and transport, Inter. J. Mult. Comput. Eng., 6 (2008), 87-101.

[31] S. Sun, J. Moortgat and A. Firoozabadi, Compositional modeling of three-phase flow with gravity using higher-order finite element methods, Water Resour. Res., 47 (2011), W05511.

[32] S. Sun and M.F. Wheeler, Symmetric and nonsymmetric discontinuous galerkin methods for reactive transport in porous media, SIAM J. Numer. Anal., 43 (2005), 195-219.

[33] S. Sun and M.F. Wheeler, Local problem-based a posteriori error estimators for discontinuous galerkin approximations of reactive transport, Comput. Geosci., 11 (2007), 87-101.

[34] Z. Sun, A second-order accurate linearized difference scheme for the two-dimensional Cahn-Hilliard equation, Math. Comp., 64 (1995), 1463-1471.

[35] T. Tang and J. Yang, Implicit-explicit scheme for the Allen-Cahn equation preserves the maximum principle, J. Comput. Math., 34 (2016), 451-461.

[36] J.D. van der Waals, The thermodynamic theory of capillarity under the hypothesis of a continuous density variation, J. Stat. Phys., 20 (1979), 200-244.

[37] C. Wang, X. Wang and S.M. Wise, Unconditionally stable schemes for equations of thin film epitaxy, Disc. Cont. Dyn. Sys. A, 28 (2010), 405-423.

[38] Z. Zhang and Z. Qiao, An adaptive time-stepping strategy for the Cahn-Hilliard equation, Commun. Comput. Phys., 11 (2012), 1261-1278.

[39] H. Zhong, X.P. Wang, A. Salama and S. Sun, Quasistatic analysis on configuration of two-phase flow in y-shaped tubes, Comput. Math. Appl., 68 (2014), 1905-1914. 